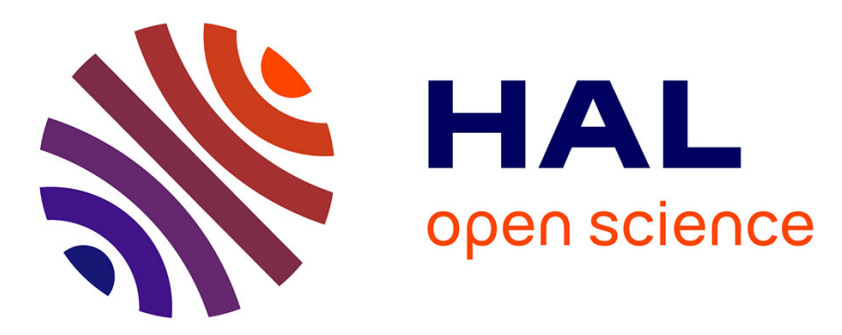

\title{
Analytical approximations of BSDEs with non-smooth driver
}

Emmanuel Gobet, Stefano Pagliarani

\section{To cite this version:}

Emmanuel Gobet, Stefano Pagliarani. Analytical approximations of BSDEs with non-smooth driver. 2014. hal-01003913

\section{HAL Id: hal-01003913 https://hal.science/hal-01003913}

Preprint submitted on 10 Jun 2014

HAL is a multi-disciplinary open access archive for the deposit and dissemination of scientific research documents, whether they are published or not. The documents may come from teaching and research institutions in France or abroad, or from public or private research centers.
L'archive ouverte pluridisciplinaire HAL, est destinée au dépôt et à la diffusion de documents scientifiques de niveau recherche, publiés ou non, émanant des établissements d'enseignement et de recherche français ou étrangers, des laboratoires publics ou privés. 


\title{
Analytical approximations of BSDEs with non-smooth driver
}

\author{
Emmanuel Gobet ${ }^{1}$, Stefano Pagliarani ${ }^{2}$ \\ Centre de Mathématiques Appliquées, Ecole Polytechnique and CNRS, Route de Saclay, 91128 Palaiseau \\ Cedex, France
}

\begin{abstract}
We provide and analyse analytical approximations of BSDEs in the limit of small nonlinearity and short time, in the case of non-smooth drivers. We identify the first and the second order approximations within this asymptotics and consider two topical financial applications: the two interest rates problem and the Funding Value Adjustment. In high dimensional diffusion setting, we show how to compute explicitly the first order formula by taking advantage of recent proxy techniques. Numerical tests up to dimension 10 illustrate the efficiency of the numerical schemes.
\end{abstract}

Keywords: backward stochastic differential equation, asymptotic expansion 2010 MSC: 65C30, 34E10

\section{Introduction}

\subsection{Model and motivation}

During the two last decades, the field of Backward Stochastic Differential Equations (BSDEs for short) has been very actively developed and widened with many respects, from theory to numerics through applications. Due to the tremendous amount of literature on the subject, it is somehow vain to be exhaustive in the references. Therefore, we simply refer the reader to EPQ97, EHM08, PR14 for theory and applications to mathematical finance, stochastic control and semi-linear PDEs, and to the recent works GT13a, GT13b. (and references therein) for fully implementable Monte-Carlo schemes.

In this work, we consider the quite standard setting of BSDE with Lipschitz driver in the natural filtration of the $q$-dimensional Brownian motion $W$, by considering $\left(Y^{\varepsilon}, Z^{\varepsilon}\right)$ the solution to

$$
\left\{\begin{aligned}
-\mathrm{d} Y_{t}^{\varepsilon} & =\left(\alpha_{t}+\beta_{t} Y_{t}^{\varepsilon}+\gamma_{t} \cdot Z_{t}^{\varepsilon}+\varepsilon g\left(t, Y_{t}^{\varepsilon}, Z_{t}^{\varepsilon}\right)\right) \mathrm{d} t-Z_{t}^{\varepsilon} \mathrm{d} W_{t}, \quad 0 \leq t<T \\
Y_{T}^{\varepsilon} & =\xi
\end{aligned}\right.
$$

In the above, $\xi$ is a random variable at a fixed terminal time $T>0, \gamma$ and $Z$ are processes taking values in $\mathbb{R}^{q}$ (written as row vectors), $g$ is Lipschitz continuous in $(y, z)$ and $\alpha, \beta, \gamma$ satisfy growth/boundedness conditions, ensuring that the driver

$$
f^{\varepsilon}(\omega, t, y, z):=\alpha_{t}+\beta_{t} y+\gamma_{t} \cdot z+\varepsilon g(t, y, z)
$$

\footnotetext{
${ }^{1}$ emmanuel.gobet@polytechnique.edu.

${ }^{2}$ pagliarani@cmap.polytechnique.fr.
} 
satisfies the conditions of the usual Lipschitz setting. These assumptions are stated in greater details later. The parameter $\varepsilon$ is small, and for convenience, we assume from now on that $\varepsilon \in(0,1]$.

If $\varepsilon=0$, the solution to (1.1) boils down to a linear BSDE which $Y$-component can be represented explicitly in terms of conditional expectation, see (1.5). Regarding applications, in mathematical finance $\left(Y^{0}, Z^{0}\right)$ is usually related to the price and hedge of the contingent claim $\xi$ using a linear pricing rule (see [EPQ97 for details). The additional non-linear term $g$ is relevant in practice when we want to go beyond the usual linear pricing rules by taking into account small market imperfections (asymmetric interest rates for lending and borrowing, funding, credit risk adjustment, etc): for related ideas, see the recent monographs Cre13, GH14. In many of these applications it is realistic to assume the parameter $\varepsilon$ determining the "size" of the non-linearity to be relatively small. For instance, in the case of the two interest rates, the role of $\varepsilon$ is played by the difference between the borrowing and the lending rates, which can be reasonably supposed to be smaller than $5 \%$. Similarly, when considering the valuation of a portfolio with Credit and Funding Value Adjustment (CVA-FVA) (accounting for provision for counterparty risk), the same role is played by the default intensity parameter, which can be estimated to be, in the most pessimistic scenarios, within $10 \%$. Our work provides explicit and computable approximations for nonlinear pricing in the limit of small non-linearity, and small time to maturity. The principle of our analysis is rather classical and relies on the knowledge of the linear solution $\left(Y^{0}, Z^{0}\right)$ (or an approximation of it). Correction terms are then computed in terms of $\left(Y^{0}, Z^{0}\right)$. The difficulty handled in our work comes from the low regularity assumptions on $g$.

\subsection{Our contribution and comparison with literature}

By taking advantage of the asymptotic property $\varepsilon \rightarrow 0$, we aim at deriving an accurate approximation of the solution, up to some order w.r.t. $\varepsilon$. Actually, in order to go further in the approximation w.r.t. $\varepsilon$, we need to identify the correction terms $\left(\frac{Y^{\varepsilon}-Y^{0}}{\varepsilon}, \frac{Z^{\varepsilon}-Z^{0}}{\varepsilon}\right)$ as $\varepsilon \rightarrow 0$. As we will see on examples (Sections 3 and 4), the proposed approximation can be computed analytically for some multidimensional problems, without any use of MonteCarlo methods. This will be achieved by incorporating proxy approximations as in GM14] or [LPP13]. The availability of analytical formulas is much interesting for having at hand a fast emulation of the solution; it could also serve as a control variate or proxy as explained in GT13b, Section 2].

The second task arising when dealing with such perturbative schemes, is the necessity to find error estimates for the approximate solutions at a given order. At the best of our knowledge, the only results that we register in this direction are those in TY13, where the authors considered the case of a smooth $\left(C^{n}\right)$ non-linearity $g$ and proved asymptotic convergence for the $n$-th order approximation, with respect to the perturbation parameter $\epsilon$. These regularity assumptions on the driver are too restrictive for many of the examples that naturally arise from financial applications, such as the ones considered in Sections 3 and 4 . Indeed, in such applications $g$ takes the form

$$
g(t, y, z):=\left(y-\eta_{t} \cdot z\right)_{+},
$$

i.e. $g$ is only piecewise differentiable. For this reason, here we do not assume that the driver $f^{\varepsilon}$ is very smooth. This is a significant difference compared to [TY13].

Had we supposed $g$ were smooth, the existence and characterization of an expansion of $\left(Y^{\varepsilon}, Z^{\varepsilon}\right)$ w.r.t. $\varepsilon$ would follow more or less directly from an iterative application of the standard differentiation result EPQ97, Proposition 2.4]. This is essentially the way followed by TY13. To account for cases like (1.3), we assume a customized weak differentiability condition on $g$ and then, we prove a first and a second order expansion w.r.t. $\varepsilon$. Another 
aspect on which our results differ from the existing literature, is that the 1-st order approximate solution is proved here to be asymptotic not only with respect to $\epsilon$ but also w.r.t. $T$, resulting in a good accuracy not only when the non-linearity is small, but also in general for short maturities. We also register additional improvements of the convergence rate for small maturities $T$ in the cases when $g$ is independent of $z$, and when $g(\omega, t, 0,0)$ is pointwise null (like for FVA in Section 4). Furthermore, these 1-st order error estimates do not only account for the asymptotics w.r.t. $\varepsilon$ and $T$ but also for the dependence on other parameters, which means they keep track of all the other model coefficients $(\alpha, g$ and $\xi)$. Finally, we also notice that we do not assume any Markovian assumptions (as a difference with TY13] where PDE arguments are used).

\subsection{Organization of the paper}

At the end of this section, we give the notations and state the assumptions used throughout the paper. In Section 2, we state our main result, by starting with formal expansion and then concluding with rigorous derivation. Section 3 is devoted to the application to pricing with two interest rates. Section 4 handles the pricing with provision for counterparty risk. Numerical experiments illustrating the excellent performance of the approximation are provided for each application above.

\subsection{Notations and assumptions}

Probability space.. Let $T>0$ and $(\Omega, \mathcal{F}, \mathbb{P})$ be a filtered probability space supporting a $q$-dimensional Brownian motion $W$, equipped with the augmented Brownian filtration $\left(\mathcal{F}_{t}\right)_{0 \leq t \leq T}$. We denote by $\mathcal{P}_{T}$ the $\sigma$-algebra of predictable sets of $\Omega \times[0, T]$. Furthermore, in order to carry out our analysis we need to introduce the following normed spaces: given an Euclidean space $\mathcal{E}$, we set

- $\mathbb{L}_{T}^{2}(\mathcal{E}):=\left\{X: \Omega \rightarrow \mathcal{E}\right.$ s.t. $X$ is $\mathcal{F}_{T}$-measurable and $\left.\mathbb{E}\left[|X|^{2}\right]<\infty\right\}$, equipped with $|X|_{L_{2}}^{2}:=\mathbb{E}\left[|X|^{2}\right]$ where $|$.$| stands for the Euclidean norm;$

- $\mathbb{H}_{T}^{2}(\mathcal{E}):=\left\{\varphi \quad: \quad \Omega \times[0, T] \rightarrow \mathcal{E}\right.$ s.t. $\varphi$ is $\mathcal{P}_{T}$-measurable (predictable) and $\left.\mathbb{E}\left[\int_{0}^{T}\left|\varphi_{t}\right|^{2} \mathrm{~d} t\right]<\infty\right\}$, equipped with the norm $\|\varphi\|^{2}:=\mathbb{E}\left[\int_{0}^{T}\left|\varphi_{t}\right|^{2} \mathrm{~d} t\right] ;$

- for $\varphi \in \mathbb{H}_{T}^{2}(\mathcal{E})$, we set $\|\varphi\|_{\theta}^{2}:=\mathbb{E}\left[\int_{0}^{T} e^{\theta t}\left|\varphi_{t}\right|^{2} \mathrm{~d} t\right]$ for a scalar $\theta \in \mathbb{R}$;

- for a bounded process $\varphi$, we set $|\varphi|_{\infty}:=\sup _{(\omega, t) \in \Omega \times[0, T]}\left|\varphi_{t}(\omega)\right|$.

We have $\|\|=.\|\cdot\|_{0}$. In our analysis, the Euclidean space $\mathcal{E}$ will be $\mathbb{R}$ or $\mathbb{R}^{q}$; since it will be clear from the context of use, we do not refer to $\mathcal{E}$ in the notation of the norm $\|\cdot\|_{\theta}$. Unless necessary for the sake of clarity, the $q$-dimensional Euclidean space will be indifferently denoted by $\mathbb{R}^{q}$ and $\left(\mathbb{R}^{q}\right)^{\top}$, whatever vectors are written as columns or rows respectively.

BSDE assumptions. The three following assumptions are considered throughout the paper.

H.1 the terminal value $\xi \in \mathbb{L}_{T}^{2}(\mathbb{R})$;

H.2 the constant part of the driver $\alpha$ belongs to $\mathbb{H}_{T}^{2}(\mathbb{R})$; the linear coefficients $\beta \in \mathbb{H}_{T}^{2}(\mathbb{R})$ and $\gamma \in \mathbb{H}_{T}^{2}\left(\mathbb{R}^{q}\right)$ are bounded processes; the non linear part $g: \Omega \times[0, T] \times \mathbb{R} \times \mathbb{R}^{q} \rightarrow \mathbb{R}$ is $\mathcal{P}_{T} \otimes \mathcal{B} \otimes \mathcal{B}^{q}$-measurable $\left(\mathcal{B}\right.$ and $\mathcal{B}^{q}$ are the Borel sigma-fields on $\mathbb{R}$ and $\mathbb{R}^{q}$ ) and $g(\cdot, \cdot, 0,0) \in \mathbb{H}_{T}^{2}(\mathbb{R})$

H.3 $g(\omega, t, \cdot, \cdot)$ is globally Lipschitz, uniformly with respect to $(\omega, t) \in \Omega \times[0, T]$, i.e. there exists $C_{g}>0$ such that, $\mathrm{d} \mathbb{P} \otimes \mathrm{d} t$-a.s.,

$$
\left|g\left(\omega, t, y_{1}, z_{1}\right)-g\left(\omega, t, y_{2}, z_{2}\right)\right| \leq C_{g}\left(\left|y_{1}-y_{2}\right|+\left|z_{1}-z_{2}\right|\right), \quad y_{1}, y_{2} \in \mathbb{R}, z_{1}, z_{2} \in \mathbb{R}^{q} .
$$


Then under the hypothesis H.1-H.3, the driver $f^{\varepsilon}(\omega, t, y, z)$ (defined in $(1.2)$ ) is globally Lipschitz in $(y, z)$, uniformly with respect to $(\omega, t) \in \Omega \times[0, T]$, with Lipschitz constant bounded by

$$
C_{f}:=|\beta|_{\infty}+|\gamma|_{\infty}+C_{g} .
$$

Thus (see [EPQ97, Theorem 2.1]), the equation (1.1) admits a unique solution $\left(Y^{\varepsilon}, Z^{\varepsilon}\right)$ in $\mathbb{H}_{T}^{2}(\mathbb{R}) \times \mathbb{H}_{T}^{2}\left(\mathbb{R}^{q}\right)$, for any $\varepsilon$. The solution $\left(Y^{0}, Z^{\sigma}\right)$ at $\varepsilon=0$ plays an important role in our analysis. In fact, (1.1) is a linear BSDE at $\varepsilon=0$, leading to the explicit representation of $Y^{0}$ (see [EPQ97, Proposition 2.2]):

$$
Y_{t}^{0}=\mathbb{E}\left[\xi M_{T}^{t}+\int_{t}^{T} M_{s}^{t} \alpha_{s} \mathrm{~d} s \mid \mathcal{F}_{t}\right]
$$

where the process $\left(M_{s}^{t}\right)_{t \leq s \leq T}$ is the exponential

$$
M_{s}^{t}=\exp \left(\int_{t}^{s}\left(\beta_{r}-\frac{1}{2}\left|\gamma_{r}\right|^{2}\right) \mathrm{d} r+\int_{t}^{s} \gamma_{r} \mathrm{~d} W_{r}\right)
$$

Besides, since $Z^{0}$ is related to the predictable representation of the martingale part of $Y^{0}$, it can be expressed using Malliavin calculus, see for instance [HNS11] we will not develop further in this direction. For convenience, we set

$$
g_{t}^{0}:=g\left(t, Y_{t}^{0}, Z_{t}^{0}\right) .
$$

The subsequent expansion of $\left(Y^{\varepsilon}, Z^{\varepsilon}\right)$ is achieved by assuming an appropriate (weak) regularity condition on $g$, namely Gateaux-differentiability, which enables us in Theorem 2.5 to establish an expansion to the second order.

H.4 For any $(u, v) \in \mathbb{H}_{T}^{2}(\mathbb{R}) \times \mathbb{H}_{T}^{2}\left(\left(\mathbb{R}^{q}\right)^{\top}\right)$, there exist $\kappa=\kappa(u, v) \in[0,1)$ and $D g^{0}(u, v) \in$ $\mathbb{H}_{T}^{2}(\mathbb{R})$ such that

$$
\mathbb{E} \int_{0}^{T}\left|\frac{g\left(t, Y_{t}^{0}+\varepsilon u_{t}, Z_{t}^{0}+\varepsilon v_{t}\right)-g_{t}^{0}-\varepsilon D g_{t}^{0}(u, v)}{\varepsilon}\right|^{2} \mathrm{~d} t=o\left(\varepsilon^{2 \kappa}\right)
$$

as $\varepsilon \rightarrow 0^{+}$.

This assumption reads as a Gateaux-differentiability condition in $L_{2}(\mathrm{dP} \otimes \mathrm{d} t)$. For cases of the form (1.3), we can verify the assumption H.4. The proof is postponed to Appendix.

Proposition 1.1. If $g(t, y, z):=\left(\eta_{t}^{Y} y+\eta_{t}^{Z} \cdot z\right)_{+}$for two bounded predictable processes $\left(\eta^{Y}, \eta^{Z}\right)$ taking values in $\mathbb{R}$ and $\left(\mathbb{R}^{q}\right)^{\top}$, then $\mathbf{H . 4}$ is satisfied with $\kappa=0$ and

$$
D g_{t}^{0}(u, v)=\left(\eta_{t}^{Y} u_{t}+\eta_{t}^{Z} \cdot v_{t}\right) \mathbb{1}_{G_{t}^{0}>0}+\left(\eta_{t}^{Y} u_{t}+\eta_{t}^{Z} \cdot v_{t}\right)_{+} \mathbb{1}_{G_{t}^{0}=0}, \quad G_{t}^{0}:=\eta_{t}^{Y} Y_{t}^{0}+\eta_{t}^{Z} \cdot Z_{t}^{0},
$$

for any $(u, v) \in \mathbb{H}_{T}^{2}(\mathbb{R}) \times \mathbb{H}_{T}^{2}\left(\left(\mathbb{R}^{q}\right)^{\top}\right)$.

Miscellaneous. For a smooth function $\varphi: \mathbb{R}^{d_{1}} \rightarrow \mathbb{R}^{d_{2}}$, we denote by $\nabla \varphi$ the gradient of $g$ which is a $d_{2} \times d_{1}$-matrix. In particular, if $\varphi$ takes scalar values, $\nabla \varphi$ is a row vector. To write the gradient w.r.t. variables $y$ or $z$, we may write $\nabla_{y} \varphi$ and $\nabla_{z} \varphi$.

Limits w.r.t. $\varepsilon \rightarrow 0$ are taken along strictly positive values of $\varepsilon \in(0,1]$.

We set

$$
C_{f^{0}}:=|\beta|_{\infty}+|\gamma|_{\infty}
$$

which is an upper bound for the Lipschitz constant of $f^{0}$. 


\section{Main results}

\subsection{Formal expansion}

For the sake of clarity, we first give heuristics by carrying out a formal expansion for the solution $\left(Y^{\varepsilon}, Z^{\varepsilon}\right)$ up to some order $n \in \mathbb{N} \backslash\{0\}$, i.e.

$$
\left(Y_{t}^{\varepsilon}, Z_{t}^{\varepsilon}\right) \approx\left(Y_{t}^{0}, Z_{t}^{0}\right)+\sum_{k=1}^{n} \frac{\varepsilon^{k}}{k !}\left(Y_{t}^{(k)}, Z_{t}^{(k)}\right), \quad\left(Y^{(k)}, Z^{(k)}\right):=\left.\frac{\mathrm{d}^{k}}{\mathrm{~d} \varepsilon^{k}}\left(Y_{t}^{\varepsilon}, Z_{t}^{\varepsilon}\right)\right|_{\varepsilon=0},
$$

omitting the issues about the possible irregularity of $g$. Namely, if $g$ is smooth enough w.r.t. $(y, z)$, the solution $\left(Y^{\varepsilon}, Z^{\varepsilon}\right)$ is differentiable w.r.t. $\varepsilon$ using the differentiability result of [EPQ97, Proposition 2.4]. The BSDE for the derivative is obtained by differentiating the initial BSDE and applying the chain rule: for $k \geq 1$,

$$
\left\{\begin{aligned}
-\mathrm{d} Y_{t}^{(k)} & =\left(\beta_{t} Y_{t}^{(k)}+\gamma_{t} \cdot Z_{t}^{(k)}+\left.k \frac{\mathrm{d}^{k-1}}{\mathrm{~d} \varepsilon^{k-1}} g\left(t, Y_{t}^{\varepsilon}, Z_{t}^{\varepsilon}\right)\right|_{\varepsilon=0}\right) \mathrm{d} t-Z_{t}^{(k)} \mathrm{d} W_{t}, \\
Y_{T}^{(k)} & =0 .
\end{aligned}\right.
$$

Since the above BSDE is linear, we get $Y_{t}^{(k)}=k \mathbb{E}\left[\left.\int_{t}^{T} M_{s}^{t} \frac{\mathrm{d}^{k-1}}{\mathrm{~d} \varepsilon^{k-1}} g\left(s, Y_{s}^{\varepsilon}, Z_{s}^{\varepsilon}\right)\right|_{\varepsilon=0} \mathrm{~d} s \mid \mathcal{F}_{t}\right]$, where the process $\left(M_{s}^{t}\right)_{t \leq s \leq T}$ is the exponential given in (1.6).

The case of non-smooth $g$. Under our assumptions, it is not possible to derive the a.s. differentiation as in EPQ97, Proposition 2.4]. Instead, we define $\left(Y^{(1)}, Z^{(1)}\right)$ and $\left(Y^{(2)}, Z^{(2)}\right)$, being inspired by what the derivatives of $\left.\frac{\mathrm{d}^{k-1}}{\mathrm{~d} \varepsilon^{k-1}} g\left(s, Y_{s}^{\varepsilon}, Z_{s}^{\varepsilon}\right)\right|_{\varepsilon=0}$ should be in the smooth case, and then we directly prove the asymptotic approximation at order $n=1$ and $n=2$, with some error estimates of the residual (Theorem 2.5). To this purpose we set, by definition,

$$
\begin{aligned}
& \left\{\begin{aligned}
-\mathrm{d} Y_{t}^{(1)} & =\left(\beta_{t} Y_{t}^{(1)}+\gamma_{t} \cdot Z_{t}^{(1)}+g_{t}^{0}\right) \mathrm{d} t-Z_{t}^{(1)} \mathrm{d} W_{t}, \\
Y_{T}^{(1)} & =0,
\end{aligned}\right. \\
& \left\{\begin{aligned}
-\mathrm{d} Y_{t}^{(2)} & =\left(\beta_{t} Y_{t}^{(2)}+\gamma_{t} \cdot Z_{t}^{(2)}+2 D g_{t}^{0}\left(Y^{(1)}, Z^{(1)}\right)\right) \mathrm{d} t-Z_{t}^{(2)} \mathrm{d} W_{t}, \\
Y_{T}^{(2)} & =0 .
\end{aligned}\right.
\end{aligned}
$$

We first state that the solutions to the above equations are well-defined in appropriate $L_{2}$-spaces.

Proposition 2.2. Under assumptions H.1-H.3 the linear BSDE 2.1] has a unique solution $\left(Y^{(1)}, Z^{(1)}\right)$ in $\mathbb{H}_{T}^{2}(\mathbb{R}) \times \mathbb{H}_{T}^{2}\left(\mathbb{R}^{q}\right)$. Furthermore, under the additional hypothesis $\mathbf{H . 4}$ the linear $B S D E 2.2$ has a unique solution $\left(Y^{(2)}, Z^{(2)}\right)$ in $\mathbb{H}_{T}^{2}(\mathbb{R}) \times \mathbb{H}_{T}^{2}\left(\mathbb{R}^{q}\right)$.

Proof. By Hypothesis H.3 on $g$ we have $\left|g\left(t, Y_{t}^{0}, Z_{t}^{0}\right)-g(t, 0,0)\right| \leq C_{g}\left(\left|Y_{t}^{0}\right|+\left|Z_{t}^{0}\right|\right), \mathrm{d} \mathbb{P} \otimes$ $\mathrm{d} t$-a.s., and since $\left(Y^{0}, Z^{0}\right) \in \mathbb{H}_{T}^{2}(\mathbb{R}) \times \mathbb{H}_{T}^{2}\left(\mathbb{R}^{q}\right)$, we have $\left(g\left(t, Y_{t}^{0}, Z_{t}^{0}\right)-g(t, 0,0)\right)_{0 \leq t \leq T} \in$ $\mathbb{H}_{T}^{2}(\mathbb{R})$. Plus, by Hypothesis H.1 $(g(t, 0,0))_{0 \leq t \leq T} \in \mathbb{H}_{T}^{2}(\mathbb{R})$, whence $\left(g_{t}^{0}=g\left(t, Y_{t}^{0}, Z_{t}^{0}\right)\right)_{0 \leq t \leq T} \in$ $\mathbb{H}_{T}^{2}(\mathbb{R})$. In addition the driver $(y, z) \mapsto \beta_{t} y+\gamma_{t} \cdot z+g_{t}^{0}$ is globally Lipschitz in $(y, z)$ since $\beta$ and $\gamma$ are bounded. In conclusion [EPQ97, Proposition 2.2], there is a unique solution $\left(Y^{(1)}, Z^{(1)}\right)$ in $\mathbb{H}_{T}^{2}(\mathbb{R}) \times \mathbb{H}_{T}^{2}\left(\mathbb{R}^{q}\right)$ as advertised.

The same arguments apply to $\left(Y^{(2)}, Z^{(2)}\right)$, mainly because $\left(D g_{t}^{0}\left(Y^{(1)}, Z^{(1)}\right)\right)_{0 \leq t \leq T} \in$ $\mathbb{H}_{T}^{2}(\mathbb{R})$, in view of the previous estimates and of Hypothesis H.4.

Last but not least, the $Y$-components of these linear BSDEs can be represented as expectations EPQ97, Proposition 2.2], similarly to 1.5. 
Corollary 2.3. Under assumptions H.1-H.3, we have

$$
Y_{t}^{(1)}=\mathbb{E}\left[\int_{t}^{T} M_{s}^{t} g_{s}^{0} \mathrm{~d} s \mid \mathcal{F}_{t}\right] .
$$

Furthermore, under the additional hypothesis H.4, we have

$$
Y_{t}^{(2)}=2 \mathbb{E}\left[\int_{t}^{T} M_{s}^{t} D g_{s}^{0}\left(Y^{(1)}, Z^{(1)}\right) \mathrm{d} s \mid \mathcal{F}_{t}\right] .
$$

\subsection{Asymptotic convergence}

In this section we present a rigorous analysis of the perturbative method introduced in the previous section. We are now in the position to prove the convergence for our 1-st and 2-nd order approximations of the true solution $\left(Y^{\varepsilon}, Z^{\varepsilon}\right)$ of BSDE (1.1), defined respectively as

$$
\begin{aligned}
& \left(\bar{Y}^{\varepsilon, 1}, \bar{Z}^{\varepsilon, 1}\right):=\left(Y^{0}, Z^{0}\right)+\varepsilon\left(Y^{(1)}, Z^{(1)}\right), \\
& \left(\bar{Y}^{\varepsilon, 2}, \bar{Z}^{\varepsilon, 2}\right):=\left(Y^{0}, Z^{0}\right)+\varepsilon\left(Y^{(1)}, Z^{(1)}\right)+\frac{\varepsilon^{2}}{2}\left(Y^{(2)}, Z^{(2)}\right) .
\end{aligned}
$$

We have the following global error bounds for the 1-st order expansion $\left(\bar{Y}^{\varepsilon, 1}, \bar{Z}^{\varepsilon, 1}\right)$.

Theorem 2.4. Under assumptions H.1-H.3 there exists a positive finite function $K($.$) ,$ increasing in all its variables, such that

$$
\begin{aligned}
& \sup _{0 \leq t \leq T}\left|Y_{t}^{\varepsilon}-\bar{Y}_{t}^{\varepsilon, 1}\right|_{L_{2}}^{2}+\left\|Z^{\varepsilon}-\bar{Z}^{\varepsilon, 1}\right\|^{2} \\
& \leq K\left(T, C_{f^{0}}, C_{f}, C_{g},|\xi|_{L_{2}}^{2},\|\alpha\|,|g(\cdot, 0,0)|_{L_{2}}^{2}\right) \varepsilon^{4}\left(\bar{C}_{g} T^{2}+|g(\cdot, 0,0)|_{L_{2}}^{2} T^{3}+T^{4}\right),
\end{aligned}
$$

where $\bar{C}_{g}$ is equal to 0 if $g$ does not depend on $z$, and 1 otherwise.

As usually in this kind of a priori error estimates for BSDEs, we can prove that the same rates are valid when the sup is inside the expectation (i.e. $\left.\mathbb{E}\left(\sup _{0 \leq t \leq T}\left|Y_{t}^{\varepsilon}-\bar{Y}_{t}^{\varepsilon, 1}\right|^{2}\right)\right)$. We leave the details to the reader.

These estimates show that we can expect accuracy of our approximation not only as $\varepsilon \rightarrow 0$, but also as $T$ is small. This is coherent with our observations in the subsequent numerical experiments. Even though it is a different phenomenon, the asymptotic behavior in the $T$ variable for approximate solutions obtained via perturbative schemes, has been already observed for some classes of analytical expansions for linear problems with variable coefficients, such as in PP14, BG12].

Note also that the rate of convergence for small maturities increase from $T^{2}$ to $T^{3}$ if $g$ does not depend on $z$, and it goes up to $T^{4}$ if we also have $g(\cdot, 0,0)=0 \mathrm{~d} \mathbb{P} \otimes \mathrm{d} t$-almost surely. This is for instance the case of the application presented in Section 4 . The improvement in the case when $g$ is independent of $z$ is not surprising since $Z$ is a gradient (from the PDE point of view) and its control is more demanding.

Proof. We make use of some well-known a priori estimates recalled in Proposition A.1 in Appendix A.1. consider $\left(Y^{0}, Z^{0}\right)$ for the first BSDE which driver $f^{0}$ (see (1.2)) has Lipschitz constant bounded by $C_{f^{\circ}}$ (defined in $(1.8)$ ), and $(0,0)$ for the second one. Then, the choice $\mu=1, \lambda^{2}=C_{f^{0}}+1$ and $\theta=C_{f^{0}}^{2}+3 C_{f^{0}}+1$ yields

$$
\left(\sup _{0 \leq t \leq T} \mathbb{E}\left|Y_{t}^{0}\right|^{2}\right) \vee\left(\left(1+C_{f^{0}}\right)^{-1} \mathbb{E} \int_{0}^{T}\left|Z_{t}^{0}\right|^{2} \mathrm{~d} t\right) \leq e^{\left(C_{f^{0}}^{2}+3 C_{f^{0}}+1\right) T}\left(\mathbb{E}|\xi|^{2}+\mathbb{E} \int_{0}^{T}\left|\alpha_{t}\right|^{2} \mathrm{~d} t\right) .
$$


Moreover, $\left(Y^{(1)}, Z^{(1)}\right)$ solves a BSDE (2.1), also with Lipschitz constant bounded by $C_{f^{0}}$. Proposition A.1. now applied with $\mu=1 / \sqrt{T}, \lambda^{2}=C_{f^{0}}+1$ and $\theta=C_{f^{0}}^{2}+3 C_{f^{0}}+1 / T$ gives

$$
\begin{aligned}
& \left(\sup _{0 \leq t \leq T} \mathbb{E}\left|Y_{t}^{(1)}\right|^{2}\right) \vee\left(\left(1+C_{f^{0}}\right)^{-1} \mathbb{E} \int_{0}^{T}\left|Z_{t}^{(1)}\right|^{2} \mathrm{~d} t\right) \\
& \leq e^{\left(C_{f^{0}}^{2}+3 C_{f^{0}}\right) T+1} T \mathbb{E} \int_{0}^{T}\left|g_{t}^{0}\right|^{2} \mathrm{~d} t
\end{aligned}
$$

(by assumption H.3, combined with (2.6))

$$
\begin{aligned}
\leq & 3 e^{\left(C_{f^{0}}^{2}+3 C_{f^{0}}\right) T+1} T\left(\mathbb{E} \int_{0}^{T}|g(t, 0,0)|^{2} \mathrm{~d} t\right. \\
& \left.+C_{g}^{2}\left(T+\bar{C}_{g}\left(1+C_{f^{0}}\right)\right) e^{\left(C_{f^{0}}^{2}+3 C_{f^{0}}+1\right) T}\left(\mathbb{E}|\xi|^{2}+\mathbb{E} \int_{0}^{T}\left|\alpha_{t}\right|^{2} \mathrm{~d} t\right)\right),
\end{aligned}
$$

where we have set $\bar{C}_{g}=0$ if $g$ does not depend on $z$, and $\bar{C}_{g}=1$ otherwise. Now, by definition 2.3, together with 2.1) and (1.1) with $\varepsilon=0$, we obtain that $\left(\bar{Y}^{\varepsilon, 1}, \bar{Z}^{\varepsilon, 1}\right)$ is the unique solution in $\mathbb{H}_{T}^{2}(\mathbb{R}) \times \mathbb{H}_{T}^{2}\left(\mathbb{R}^{q}\right)$ of

$$
\left\{\begin{aligned}
-\mathrm{d} \bar{Y}_{t}^{\varepsilon, 1} & =\left(\alpha_{t}+\beta_{t} \bar{Y}_{t}^{\varepsilon, 1}+\gamma_{t} \cdot \bar{Z}_{t}^{\varepsilon, 1}+\varepsilon g_{t}^{0}\right) \mathrm{d} t-\bar{Z}_{t}^{\varepsilon, 1} \mathrm{~d} W_{t}, \\
\bar{Y}_{T}^{\varepsilon, 1} & =\xi .
\end{aligned}\right.
$$

Again, we make use of the a priori estimates of Proposition A.1. by taking $\left(Y^{\varepsilon}, Z^{\varepsilon}\right)$ for the first BSDE (with Lipschitz constant bounded by $C_{f}$, see $(1.4)$ ) and $\left(\bar{Y}^{\varepsilon, 1}, \bar{Z}^{\varepsilon, 1}\right)$ for the second one, and with the following choice of the parameters: $\mu=1 / \sqrt{T}, \lambda^{2}=C_{f}+1$ and $\theta=C_{f}^{2}+3 C_{f}+1 / T$. We obtain

$$
\sup _{0 \leq t \leq T} \mathbb{E}\left|Y_{t}^{\varepsilon}-\bar{Y}_{t}^{\varepsilon, 1}\right|^{2}+\mathbb{E} \int_{0}^{T}\left|Z_{t}^{\varepsilon}-\bar{Z}_{t}^{\varepsilon, 1}\right|^{2} \mathrm{~d} t \leq\left(2+C_{f}\right) e^{\left(C_{f}^{2}+3 C_{f}\right) T+1} T \mathbb{E} \int_{0}^{T}\left|\delta_{t}^{\varepsilon, 1}\right|^{2} \mathrm{~d} t,
$$

with

$$
\delta_{t}^{\varepsilon, 1}=\epsilon\left(g\left(t, \bar{Y}_{t}^{\varepsilon, 1}, \bar{Z}_{t}^{\varepsilon, 1}\right)-g\left(t, Y_{t}^{0}, Z_{t}^{0}\right)\right) .
$$

By the Lipschitzianity of $g$ (Hypothesis H.3) we have

$$
\left|\delta_{t}^{\varepsilon, 1}\right| \leq \epsilon C_{g}\left(\left|\bar{Y}_{t}^{\varepsilon, 1}-Y_{t}^{0}\right|+\bar{C}_{g}\left|\bar{Z}_{t}^{\varepsilon, 1}-Z_{t}^{0}\right|\right)=\epsilon^{2} C_{g}\left(\left|Y_{t}^{(1)}\right|+\bar{C}_{g}\left|Z_{t}^{(1)}\right|\right),
$$

and thus, by combining (2.8) with 2.9 -2.7 we get

$$
\begin{aligned}
& \sup _{0 \leq t \leq T}\left|Y_{t}^{\varepsilon}-\bar{Y}_{t}^{\varepsilon, 1}\right|_{L_{2}}^{2}+\left\|Z^{\varepsilon}-\bar{Z}^{\varepsilon, 1}\right\|^{2} \\
& \leq\left(2+C_{f}\right) \epsilon^{4} C_{g}^{2} e^{\left(C_{f}^{2}+3 C_{f}\right) T+1} T \mathbb{E} \int_{0}^{T}\left(\left|Y_{t}^{(1)}\right|+\bar{C}_{g}\left|Z_{t}^{(1)}\right|\right)^{2} \mathrm{~d} t \\
& \leq\left(2+C_{f}\right) \epsilon^{4} C_{g}^{2} e^{\left(C_{f}^{2}+3 C_{f}\right) T+1} 2 T\left(T+\bar{C}_{g}\left(1+C_{f^{0}}\right)\right) 3 e^{\left(C_{f^{0}}^{2}+3 C_{f^{0}}\right) T+1} T\left(\mathbb{E} \int_{0}^{T}|g(t, 0,0)|^{2} \mathrm{~d} t\right. \\
& \left.\quad+C_{g}^{2}\left(T+\bar{C}_{g}\left(1+C_{f^{0}}\right)\right) e^{\left(C_{f^{0}}^{2}+3 C_{f^{0}}+1\right) T}\left(\mathbb{E}|\xi|^{2}+\mathbb{E} \int_{0}^{T}\left|\alpha_{t}\right|^{2} \mathrm{~d} t\right)\right),
\end{aligned}
$$

which concludes the proof. 
We now consider the 2-nd order expansion $\left(\bar{Y}^{\varepsilon, 1}, \bar{Z}^{\varepsilon, 1}\right)$. In this case it is possible to prove asymptotic estimates with respect to the perturbation parameter $\epsilon$, but not with respect to the time $T$, due the fact we have no information about the rate of convergence of the integral (1.7) in Hypothesis $\mathbf{H . 4}$ as $T$ goes to 0. We have the following result.

Theorem 2.5. Under assumptions H.1-H.4 we have

$$
\sup _{0 \leq t \leq T} \mathbb{E}\left|Y_{t}^{\varepsilon}-\bar{Y}_{t}^{\varepsilon, 2}\right|^{2}+\left\|Z^{\varepsilon}-\bar{Z}^{\varepsilon, 2}\right\|^{2}=o\left(\varepsilon^{4+2 \kappa}\right) \quad \text { as } \varepsilon \rightarrow 0
$$

where $\kappa$ is the exponent associated to Hypothesis $\mathbf{H . 4}$ with $(u, v)=\left(Y^{(1)}, Z^{(1)}\right)$.

Proof. By definition (2.4), along with 2.1, 2.2 and 1.1 with $\varepsilon=0$, we obtain that $\left(\bar{Y}^{\varepsilon, 2}, \bar{Z}^{\varepsilon, 2}\right)$ is the unique solution in $\mathbb{H}_{T}^{2}(\mathbb{R}) \times \mathbb{H}_{T}^{2}\left(\mathbb{R}^{q}\right)$ of

$$
\left\{\begin{aligned}
-\mathrm{d} \bar{Y}_{t}^{\varepsilon, 2} & =\left(\alpha_{t}+\beta_{t} \bar{Y}_{t}^{\varepsilon, 2}+\gamma_{t} \cdot \bar{Z}_{t}^{\varepsilon, 2}+\varepsilon g_{t}^{0}+\varepsilon^{2} D g_{t}^{0}\left(Y^{(1)}, Z^{(1)}\right)\right) \mathrm{d} t-\bar{Z}_{t}^{\varepsilon, 2} \mathrm{~d} W_{t}, \\
\bar{Y}_{T}^{\varepsilon, 2} & =\xi .
\end{aligned}\right.
$$

Therefore, by Proposition A.1 with the same parameters $(\mu, \lambda, \theta)$ as before, we obtain

$$
\sup _{0 \leq t \leq T} \mathbb{E}\left|Y_{t}^{\varepsilon}-\bar{Y}_{t}^{\varepsilon, 2}\right|^{2} \leq\left\|\delta^{\varepsilon, 2}\right\|_{\theta}^{2}, \quad \mathbb{E} \int_{0}^{T}\left|Z_{t}^{\varepsilon}-\bar{Z}_{t}^{\varepsilon, 2}\right|^{2} \mathrm{~d} t \leq\left(C_{f}+1\right)\left\|\delta^{\varepsilon, 2}\right\|_{\theta}^{2},
$$

with

$$
\delta_{t}^{\varepsilon, 2}=\epsilon\left(g\left(t, \bar{Y}_{t}^{\varepsilon, 2}, \bar{Z}_{t}^{\varepsilon, 2}\right)-g_{t}^{0}-\epsilon D g_{t}^{0}\left(Y^{(1)}, Z^{(1)}\right)\right) .
$$

Now, using the definitions (2.3)-2.4 and the Lipschitzianity of $g$, we write

$$
\begin{aligned}
\left|\delta_{t}^{\varepsilon, 2}\right| \leq & \varepsilon\left|g\left(t, \bar{Y}_{t}^{\varepsilon, 2}, \bar{Z}_{t}^{\varepsilon, 2}\right)-g\left(t, \bar{Y}_{t}^{\varepsilon, 1}, \bar{Z}_{t}^{\varepsilon, 1}\right)\right|+\varepsilon\left|g\left(t, \bar{Y}_{t}^{\varepsilon, 1}, \bar{Z}_{t}^{\varepsilon, 1}\right)-g_{t}^{0}-\varepsilon D g_{t}^{0}\left(Y^{(1)}, Z^{(1)}\right)\right| \\
\leq & C_{g} \frac{\varepsilon^{3}}{2}\left(\left|Y_{t}^{(2)}\right|+\left|Z_{t}^{(2)}\right|\right) \\
& +\varepsilon^{2}\left|\frac{g\left(t, Y_{t}^{0}+\varepsilon Y_{t}^{(1)}, Z_{t}^{0}+\varepsilon Z_{t}^{(1)}\right)-g\left(t, Y_{t}^{0}, Z_{t}^{0}\right)}{\varepsilon}-D g_{t}^{0}\left(Y^{(1)}, Z^{(1)}\right)\right| .
\end{aligned}
$$

In view of $\left|Y^{(2)}\right|+\left|Z^{(2)}\right| \in \mathbb{H}_{T}^{2}(\mathbb{R})$ and of Hypothesis $\mathbf{H . 4}$ with $(u, v)=\left(Y^{(1)}, Z^{(1)}\right)$, we obtain

$$
\|\delta\|_{\theta}=O\left(\varepsilon^{3}\right)+o\left(\varepsilon^{2+\kappa}\right),
$$

whence the announced result.

\section{Application to pricing and hedging under two interest rates}

\subsection{Model}

We apply our expansion technique to the non-arbitrage valuation of a contingent claim and its related hedging strategy, in the case when there are two different interest rates for borrowing and lending money. We assume a complete market model with $d$ risky assets $S^{1}, \cdots, S^{d}$, whose dynamics under the real-world probability measure $\mathbb{P}$ are given by

$$
\mathrm{d} X_{t}^{i}=\left(\mu_{t}^{i}-\frac{1}{2}\left|\sigma_{t}^{i}\right|^{2}\right) \mathrm{d} t+\sigma_{t}^{i} \mathrm{~d} W_{t}, \quad i=1, \cdots, d,
$$

where $X_{t}^{i}:=\log \left(S_{t}^{i}\right), W=\left(W^{1}, \cdots, W^{d}\right)^{\top}$ is a $d$-dimensional Brownian motion, and $\left(\sigma_{t}^{i}\right)_{0 \leq t \leq T}$ is a $\mathbb{R}^{d}$-valued stochastic process, square integrable with respect to $t$. Denoting by $\sigma=\left(\sigma_{i, j}\right)_{1 \leq i, j \leq d}$ the $d \times d$ matrix whose $i$-th row is given by the vector $\sigma^{i}$, we also assume that $\sigma$ has full rank, with $\sigma^{-1}$ being a bounded process. 
Moreover, $\left(r_{t}\right)_{0 \leq t \leq T}$ is an $\mathbb{R}_{+}$-valued bounded process representing the lending interest rate, say the log-return of a riskless bank-account from the investor point of view.

Consider now another $\mathbb{R}_{+}$-valued bounded process $\left(R_{t}\right)_{0 \leq t \leq T}$ (typically $R \geq r$ ), representing the borrowing interest rate, i.e. the cost of the money for an investor who goes short in its cash position. Consider also a financial claim whose terminal payoff at time $T$ is given by a random variable $\xi \in \mathbb{L}_{T}^{2}(\mathbb{R})$ : it can be shown (see [EPQ97]) that the terminal payoff $\xi$ can be perfectly replicated by a self-financing portfolio. Namely, if $Y_{t}$ and $\pi=\left(\pi^{1}, \cdots, \pi^{d}\right)$ represent the portfolio value and the hedging strategy (the vector of amounts in risky assets) respectively, then $(Y, \pi)$ solves the non-linear BSDE:

$$
\left\{\begin{aligned}
-\mathrm{d} Y_{t} & =\left(-r_{t} Y_{t}-\pi_{t}\left(\mu_{t}-r_{t} \mathbf{1}\right)+\left(R_{t}-r_{t}\right)\left(Y_{t}-\pi_{t} \mathbf{1}\right)_{-}\right) \mathrm{d} t-\pi_{t} \sigma_{t} \mathrm{~d} W_{t}, \quad 0 \leq t<T, \\
Y_{T} & =\xi
\end{aligned}\right.
$$

where $\mathbf{1}$ is the (column) vector whose all components are 1.

The use of our asymptotic expansion is justified by the fact that the difference between the two interest rates can be considered relatively small, i.e $\left(R_{t}-r_{t}\right) \leq 5 \%$. Thus we can assume $R_{t}=r_{t}+\epsilon D_{t}$, with $\epsilon<<1$ and $D_{t} \sim 1$. Therefore, by setting $Z_{t}=\pi_{t} \sigma_{t}$, BSDE (3.2) can be rewritten as

$$
\left\{\begin{aligned}
-\mathrm{d} Y_{t} & =\left(-r_{t} Y_{t}-Z_{t} \sigma_{t}^{-1}\left(\mu_{t}-r_{t} \mathbf{1}\right)+\epsilon D_{t}\left(Y_{t}-Z_{t} \sigma_{t}^{-1} \mathbf{1}\right)_{-}\right) \mathrm{d} t-Z_{t} \mathrm{~d} W_{t}, \quad 0 \leq t<T, \\
Y_{T} & =\xi .
\end{aligned}\right.
$$

In order to fit our initial framework in (1.1) we set $\alpha \equiv 0, \beta_{t}=-r_{t}, \gamma_{t}=-\left[\sigma_{t}^{-1}\left(\mu_{t}-r_{t} \mathbf{1}\right)\right]^{\top}$ and $g(t, y, z)=D_{t}\left(y-z \sigma_{t}^{-1} \mathbf{1}\right)_{-}=D_{t}\left(z \sigma_{t}^{-1} \mathbf{1}-y\right)_{+}$. Furthermore, this model satisfies Hypothesis H.1-H.4 (see Proposition 1.1) with

$$
D g_{t}^{0}(u, v)=D_{t}\left[\left(v_{t} \sigma_{t}^{-1} \mathbf{1}-u_{t}\right) \mathbb{1}_{Y_{t}^{0}<Z_{t}^{0} \sigma_{t}^{-1} \mathbf{1}}+\left(v_{t} \sigma_{t}^{-1} \mathbf{1}-u_{t}\right)_{+} \mathbb{1}_{Y_{t}^{0}=Z_{t}^{0} \sigma_{t}^{-1} \mathbf{1}}\right] .
$$

The proxy solution $Y^{0}$ of BSDE (3.3) is the usual no-arbitrage value of a contingent claim in a market without imperfections, with a single interest rate $r$, i.e.

$$
Y_{t}^{0}=\mathbb{E}_{\mathbb{P}}\left[e^{-\int_{t}^{T} r_{s} \mathrm{~d} s} H_{T}^{t} \xi \mid \mathcal{F}_{t}\right],
$$

with $\left(H_{s}^{t}\right)_{t \leq s \leq T}$ being the exponential martingale

$$
H_{s}^{t}=\exp \left(-\frac{1}{2} \int_{t}^{s}\left|\sigma_{\tau}^{-1}\left(\mu_{\tau}-r_{\tau} \mathbf{1}\right)\right|^{2} \mathrm{~d} \tau-\int_{t}^{s}\left(\mu_{\tau}-r_{\tau} \mathbf{1}\right)^{\top}\left[\sigma_{\tau}^{-1}\right]^{\top} \mathrm{d} W_{\tau}\right), \quad t \leq s \leq T,
$$

whereas, by Proposition 2.2 , the first correction is given by

$$
Y_{t}^{(1)}=\mathbb{E}_{\mathbb{P}}\left[\int_{t}^{T} e^{-\int_{t}^{s} r_{u} \mathrm{~d} u} H_{s}^{t} D_{s}\left(Y_{s}^{0}-Z_{s}^{0} \sigma_{s}^{-1} \mathbf{1}\right)_{-} \mathrm{d} s \mid \mathcal{F}_{t}\right] .
$$

Note that, the expectations (3.4)-(3.5) can be also expressed in terms of the risk-neutral measure $\mathbb{Q}$, defined by $\left.\frac{\mathrm{d} \mathbb{Q}}{\mathrm{d} \mathbb{P}}\right|_{\mathcal{F}_{T}}:=H_{T}^{0}$, as

$$
Y_{t}^{0}=\mathbb{E}_{\mathbb{Q}}\left[e^{-\int_{t}^{T} r_{s} \mathrm{~d} s} \xi \mid \mathcal{F}_{t}\right], \quad Y_{t}^{(1)}=\mathbb{E}_{\mathbb{Q}}\left[\int_{t}^{T} e^{-\int_{t}^{s} r_{u} \mathrm{~d} u} D_{s}\left(Y_{s}^{0}-Z_{s}^{0} \sigma_{s}^{-1} \mathbf{1}\right)_{-} \mathrm{d} s \mid \mathcal{F}_{t}\right],
$$

and the dynamics of $X$ under $\mathbb{Q}$ become

$$
\mathrm{d} X_{t}^{i}=\left(r_{t}-\frac{1}{2}\left|\sigma_{t}^{i}\right|^{2}\right) \mathrm{d} t+\sigma_{t}^{i} \mathrm{~d} W_{t}, \quad i=1, \cdots, d .
$$


In the case of Markovian dynamics, we also obtain a characterization for the terms $Z^{0}$ and $Z^{1}$ in the expansion of the hedging strategy $Z$. Consider $\sigma_{t} \equiv \sigma\left(t, X_{t}\right), r_{t} \equiv r\left(t, X_{t}\right)$, $D_{t} \equiv D(t)$ and $\xi \equiv \psi\left(X_{T}\right)$ in (3.1)-(3.3). Under mild assumptions on the coefficients and the payoff function we have the following generalized Feynman-Kac representation formula:

$$
\begin{aligned}
\left(Y_{t}^{0}, Z_{t}^{0}\right) & =\left(u_{0}\left(t, X_{t}\right), \nabla_{x} u_{0}\left(t, X_{t}\right) \sigma\left(t, X_{t}\right)\right), \\
\left(Y_{t}^{(1)}, Z_{t}^{(1)}\right) & =\left(u_{1}\left(t, X_{t}\right), \nabla_{x} u_{1}\left(t, X_{t}\right) \sigma\left(t, X_{t}\right)\right),
\end{aligned}
$$

where $u_{0}$ and $u_{1}$ are the classical solutions (unique within a certain class of functions) of

$$
\left\{\begin{array}{rlrl}
\mathcal{L} u_{0}(t, x) & =0, & 0 \leq t<T, & x \in \mathbb{R}^{d} \\
u_{0}(T, x) & =\psi(x), & x \in \mathbb{R}^{d}
\end{array}\right.
$$

and

$$
\left\{\begin{array}{rlrl}
\mathcal{L} u_{1}(t, x) & =-D(t)\left(u_{0}(t, x)-\nabla_{x} u_{0}(t, x)\right)_{-}, & 0 \leq t<T, & x \in \mathbb{R}^{d} \\
u_{1}(T, x) & =0, & x \in \mathbb{R}^{d}
\end{array}\right.
$$

respectively, and where $\mathcal{L}$ is the parabolic operator

$$
\mathcal{L}=\partial_{t}+r(t, x)\left(-1+\sum_{i=1}^{d} \partial_{x_{i}}\right)+\frac{1}{2} \sum_{i, j=1}^{d}\left(\left(\sigma \sigma^{\top}\right)_{i, j}(t, x) \partial_{x_{i}, x_{j}}-\sigma_{i, j}^{2}(t, x) \partial_{x_{i}}\right)
$$

In the next section we test the accuracy of our approximation in a particular case where it is possible to carry out a semi-explicit representation for the solution of $(3.9)$.

3.2. Example about linear combination of Call and Put options under the Black and Scholes model: numerical comparisons

Consider the case of a market with only one underlying asset following the classical Black and Scholes model $(d=q=1)$. Equation (3.6) then becomes

$$
\mathrm{d} X_{t}=\left(r-\frac{1}{2}|\sigma|^{2}\right) \mathrm{d} t+\sigma \mathrm{d} W_{t},
$$

with $\sigma>0, r \geq 0$. Assume also the borrowing interest $R=r+\epsilon$, and consider a Markovian payoff $\xi=\psi\left(X_{T}\right)$, with $\psi$ being the linear combination of $n$ Call and Put options with maturity $T$ and $\log$ strikes $\left(k_{j}\right)_{j}$, i.e.

$$
\psi(x)=\sum_{j=1}^{n}\left(c_{C, j}\left(e^{x}-e^{k_{j}}\right)_{+}+c_{P, j}\left(e^{k_{j}}-e^{x}\right)_{+}\right),
$$

with some portfolio weights $c_{C, j}, c_{P, j} \in \mathbb{R}$. Note that, in this model the operator $\mathcal{L}$ in 3.10 reduces to the heat-type (Black and Scholes) operator

$$
\mathcal{L}=\partial_{t}+r\left(-1+\partial_{x}\right)+\frac{1}{2} \sigma^{2}\left(\partial_{x, x}-\partial_{x}\right),
$$

whose fundamental solution is $e^{-r(T-t)} \Gamma(t, x ; T, y)$, where

$$
\Gamma(t, x ; T, y)=\frac{1}{\sqrt{2 \pi \sigma^{2}(T-t)}} e^{-\frac{\left(x+\left(r-\frac{\sigma^{2}}{2}\right)(T-t)-y\right)^{2}}{2 \sigma^{2}(T-t)}}
$$


is the Gaussian transition density of the process $X$. At the leading order, we obtain that $\left(Y^{0}, Z^{0}\right)$ is given by (3.7) with

$$
u_{0}(t, x)=\sum_{j=1}^{n}\left(c_{C, j} \mathrm{BS}^{C}\left(t, x ; k_{j}\right)+c_{P, j} \mathrm{BS}^{P}\left(t, x ; k_{j}\right)\right), \quad 0 \leq t<T, x \in \mathbb{R},
$$

where $\mathrm{BS}^{C}$ and $\mathrm{BS}^{P}$ stand for the classical Black and Scholes price functions of a Call and a Put option respectively, i.e. defined as follows

$$
\begin{aligned}
\mathcal{N}(x) & :=\int_{-\infty}^{x} \frac{e^{-u^{2} / 2}}{\sqrt{2 \pi}} \mathrm{d} u \\
\mathcal{N}^{+}\left(t, x ; T, k_{j}\right) & :=\int_{-\infty}^{k_{j}} \Gamma(t, x ; T, y) \mathrm{d} y=\mathcal{N}\left(\frac{k_{j}-r(T-t)-x}{\sigma \sqrt{T-t}}+\frac{1}{2} \sigma \sqrt{T-t}\right) \\
\mathcal{N}^{-}\left(t, x ; T, k_{j}\right) & :=\mathcal{N}\left(\frac{k_{j}-r(T-t)-x}{\sigma \sqrt{T-t}}-\frac{1}{2} \sigma \sqrt{T-t}\right) \\
\mathrm{BS}^{P}\left(t, x ; k_{j}\right) & :=e^{k_{j}-r(T-t)} \mathcal{N}^{+}\left(t, x ; T, k_{j}\right)-e^{x} \mathcal{N}^{-}\left(t, x ; T, k_{j}\right) \\
\mathrm{BS}^{C}\left(t, x ; k_{j}\right) & :=e^{x}-e^{k_{j}-r(T-t)}+\mathrm{BS}^{P}\left(t, x ; k_{j}\right)
\end{aligned}
$$

The first order correcting term $\left(Y_{t}^{(1)}, Z_{t}^{(1)}\right)$ is given by (3.8) with $u_{1}(t, x)$ solving (3.9), which now reduces to

$$
\left\{\begin{array}{rlrl}
\mathcal{L} u_{1}(t, x) & =-\left(u_{0}(t, x)-\partial_{x} u_{0}(t, x)\right)_{-}, & 0 \leq t<T, & x \in \mathbb{R} \\
u_{1}(T, x) & =0, & x \in \mathbb{R} .
\end{array}\right.
$$

Thus the computation of $u_{1}$ requires the identification of $u_{0}(t, x)-\partial_{x} u_{0}(t, x)$, a function which is interpreted as the amount invested in cash used to hedge the portfolio of Call and Put options. In this Black and Scholes framework, from (3.11)-(3.12) we simply deduce

$$
u_{0}(t, x)-\partial_{x} u_{0}(t, x)=\sum_{j=1}^{n} e^{k_{j}-r(T-t)}\left(c_{C, j}\left(\mathcal{N}^{+}\left(t, x ; T, k_{j}\right)-1\right)+c_{P, j} \mathcal{N}^{+}\left(t, x ; T, k_{j}\right)\right) .
$$

To compute $u_{1}$ we define the sets

$$
\Pi_{t}:=\left\{\zeta \in \mathbb{R} \mid u_{0}(t, \zeta)-\partial_{\zeta} u_{0}(t, \zeta) \leq 0\right\}, \quad 0 \leq t \leq T,
$$

and by Duhamel's principle, it leads to the solution

$$
\begin{aligned}
u_{1}(t, x)= & \int_{t}^{T} \int_{\mathbb{R}} e^{-r(s-t)} \Gamma(t, x ; s, \zeta)\left(u_{0}(s, \zeta)-\partial_{\zeta} u_{0}(s, \zeta)\right)_{-} \mathrm{d} \zeta \mathrm{d} s \\
= & -e^{-r(T-t)} \sum_{j=1}^{n}\left(c_{C, j}+c_{P, j}\right) e^{k_{j}} \int_{t}^{T} \int_{\Pi_{s}} \Gamma(t, x ; s, \zeta) \mathcal{N}^{+}\left(s, \zeta ; T, k_{j}\right) \mathrm{d} \zeta \mathrm{d} s \\
& +e^{-r(T-t)} \sum_{j=1}^{n} c_{C, j} e^{k_{j}} \int_{t}^{T} \int_{\Pi_{s}} \Gamma(t, x ; s, \zeta) \mathrm{d} \zeta \mathrm{d} s
\end{aligned}
$$

The first order derivative $\partial_{x} u_{1}$, which determines the first order correction term $Z^{(1)}$ of the hedging component $Z$, can then be obtained by a direct differentiation in $x$, leading to another space integral involving $\partial_{x} \Gamma(t, x ; s, \zeta)$. The latter and the one in (3.15) are not explicitly solvable. Nevertheless, it is possible to find an arbitrarily accurate explicit approximation of such an integral, by approximating the analytic function $\mathcal{N}$ by means of its Taylor series. 
1-st numerical test: comparison with [GLW05 . Next we test the accuracy of our 1-st order expansion $\bar{Y}_{0}^{1, \epsilon}=u_{0}\left(t, X_{0}\right)+\varepsilon u_{1}\left(t, X_{0}\right)$ for the true solution $Y_{0}$, by comparing it with the approximation proposed in GLW05, by means of a regression-based Monte Carlo algorithm (see BD07, GT13a, GT13b for more details on these algorithms). Note that the latter is a quite standard benchmark in literature for this task. According to Gobet et al., we consider the following payoff made of a linear combination of two call options with $\log$-strikes $k_{1}$ and $k_{2}$, i.e.

$$
\psi(x)=\left(e^{x}-e^{k_{1}}\right)_{+}-2\left(e^{x}-e^{k_{2}}\right)_{+},
$$

with the parameters

$r=1 \%, \quad R=6 \%, \quad \sigma=0.2, \quad t=0, \quad T=0.25, \quad X_{0}=\log 100, \quad k_{1}=\log 95, \quad k_{2}=\log 105$.

From (3.13)-(3.14), it is easy to check that $\Pi_{s}=\left(-\infty, p\left(s, T, k_{1}, k_{2}\right)\right]$ for a threshold function $p\left(s, T, k_{1}, k_{2}\right)$ which can be numerically computed (and used in (3.15)). We obtain the results reported in Table 1 .

\begin{tabular}{|c|c|c|}
\hline$Y_{0}^{\mathrm{G}}$ & $Y_{0}^{0}$ & $\bar{Y}_{0}^{1, \epsilon}$ \\
\hline 2.95 & 2.76 & 2.96 \\
\hline
\end{tabular}

Table 1: 1 -st order approximation accuracy. $Y$-value at time 0 according to the Monte Carlo simulation by Gobet et al. $\left(Y_{0}^{\mathrm{G}}\right)$, and to our 0 -th and 1-st order approximations $\left(Y_{0}^{0}\right.$ and $\bar{Y}_{0}^{1, \epsilon}$ respectively).

2-nd numerical test: comparison with BD07] . In our second test we test the accuracy of our 1-st order expansion $\bar{Y}_{t}^{1, \epsilon}$, by comparing it with the approximation proposed in [BD07, where the authors made use of a Picard scheme to simulate the BSDE (3.2). According to Bender et al., we consider the following payoff given by the sum of two Call and Put options with same log-strike $k$, i.e.

$$
\psi(x)=\left|e^{x}-e^{k}\right|=\left(e^{x}-e^{k}\right)_{+}+\left(e^{k}-e^{x}\right)_{+} .
$$

In this case one has

$$
u_{0}(s, \zeta)-\partial_{\zeta} u_{0}(s, \zeta)=e^{k-r(T-s)}\left(2 \mathcal{N}^{+}(s, \zeta ; T, k)-1\right) \leq 0 \Longleftrightarrow \zeta \geq k-\left(r-\frac{\sigma^{2}}{2}\right)(T-s),
$$

and thus we get $\Pi_{s}=\left[k-\left(r-\frac{\sigma^{2}}{2}\right)(T-s),+\infty\right)$ in 3.15). We use the same parameters as in BD07:

$$
r=1 \%, \quad R=6 \%, \quad \sigma=0.2, \quad t=0, \quad T=2, \quad X_{0}=\log 100, \quad k=\log 100,
$$

and we obtain the results reported in Table 2

\begin{tabular}{|c|c|c|}
\hline$Y_{0}^{\mathrm{B}}$ & $Y_{0}^{0}$ & $\bar{Y}_{0}^{1, \epsilon}$ \\
\hline 24.56 & 22.32 & 24.51 \\
\hline
\end{tabular}

Table 2: 1-st order approximation accuracy. $Y$-value at time 0 according to the approximation proposed by Bender et al. $\left(Y_{0}^{\mathrm{B}}\right)$, and to our 0 -th and 1-st order approximations ( $Y_{0}^{0}$ and $\bar{Y}_{0}^{1, \epsilon}$ respectively).

Conclusive remarks. In both tests, our first order approximation is very close to the reference value obtained by regression Monte-Carlo schemes, with the advantage of being very quick to compute. Because of the very good accuracy of the 1st order approximation, it seems to be not necessary to go up to the second order approximation which is more tedious to numerically compute. 


\section{Application to provision for counterparty risk}

\subsection{Model}

We apply our expansion result to compute the Funding Valuation Adjustment (FVA) with provision for counterparty risk [GH14, Section 5.8]. This is associated to the BSDE

$$
\left\{\begin{aligned}
-\mathrm{d} Y_{t} & =\left(-\lambda(1-R)\left(Y_{t}\right)_{+}-r_{t} Y_{t}\right) \mathrm{d} t-Z_{t} \mathrm{~d} W_{t}, \quad 0 \leq t<T, \\
Y_{T} & =\xi
\end{aligned}\right.
$$

where $R$ is the recovery rate, $r_{t}$ the bounded risk-free interest rate (possibly stochastic), and $\lambda$ the default intensity of the counterparty. Moreover, $W$ is a $q$ dimensional Brownian motion, whose components being the sources of randomness in the market. The model is directly given under the risk-neutral measure $\mathbb{Q}$. Since assessing the counterparty risk, $Y$ represents here the no-arbitrage value, from the investor prospective, of a global position composed of several claims exchanged with a defaultable counterpart. Therefore, we shall consider that the terminal random variable $\xi$ is a linear combination of several financial contracts, i.e.

$$
\xi=\sum_{j \in \mathcal{J}} c_{j} \Psi^{j}, \quad \Psi^{i} \in \mathbb{L}_{T}^{2}(\mathbb{R})
$$

where $\mathcal{J}$ is a countable family of indexes, and where $c_{j} \in \mathbb{R} \backslash\{0\}$ are some portfolio weights. In other words, $\Psi^{j}$ denotes the terminal value at time $T$ of the $j$-th financial claim exchanged between the investor and its counterpart. We can assume without loss of generality that $\Psi^{j} \geq \mu^{j}>0, j=1, \cdots, n$, unless to consider the decomposition

$$
\xi=\sum_{j \in \mathcal{J}} c_{j}\left(\left(\left(\Psi^{j}\right)_{+}+\mu^{j}\right)-\left(\left(\Psi^{j}\right)_{-}+\mu^{j}\right)\right), \quad \mu^{j}>0 .
$$

The need for this condition will appear more clearly in the change of numeraire of Section 4.2 .

Example 4.6. In the example of Section 4.4 each random variable $\Psi^{j}$ will represent the terminal payoff of a European type derivative with maturity $T$, written on an underlying process $S_{t}=\left(S_{t}^{1}, \ldots, S_{t}^{d}\right)$, with $d \leq q$. However, our setting is more general and allows to include the case of a pool of derivatives with different maturities $T_{j} \neq T$.

Our asymptotic method relies on $\lambda$ being suitably small; this is related to the credit spread, whose order in usual cases is few hundreds bps at most, i.e. $\lambda \leq 1-3 \%$. Our asymptotic setting seems thus to be reasonable. Then, the proxy $Y^{0}$ used for pricing is given by the usual risk-neutral price. To fit our current framework, we set $\varepsilon=\lambda, g(t, \omega, y, z)=$ $-(1-R) y_{+}, \alpha_{t} \equiv 0, \beta_{t}=-r_{t}$ and $\gamma_{t} \equiv 0$. Note also that this example fits assumptions H.1-H.4 (see Proposition 1.1) with

$$
D g_{t}^{0}(u, v)=-(1-R)\left[u_{t} \mathbb{1}_{Y_{t}^{0}>0}+\left(u_{t}\right)_{+} \mathbb{1}_{Y_{t}^{0}=0}\right] .
$$

The proxy solution $Y^{0}$ of BSDE 4.16) is given by

$$
Y_{t}^{0}=\sum_{j \in \mathcal{J}} c_{j} \Psi_{t}^{j}, \quad \Psi_{t}^{j}=\mathbb{E}\left[e^{-\int_{t}^{T} r_{s} \mathrm{~d} s} \Psi^{j} \mid \mathcal{F}_{t}\right], \quad 0 \leq t \leq T .
$$

Here $\Psi_{t}^{j}$ represents the usual no-arbitrage value at time $t$ of the contract $\Psi^{j}$ (see MR05]), computed without taking into account the possibility of default of the counterpart. In 
particular, the process $e^{-\int_{0}^{t} r_{s} \mathrm{~d} s} \Psi_{t}^{j}$ is a positive $\left(\Psi^{j} \geq \mu^{j}>0\right)$ martingale whose dynamics is given by

$$
\frac{\mathrm{d} \Psi_{t}^{j}}{\Psi_{t}^{j}}=r_{t} \mathrm{~d} t+\sigma_{t}^{j} \mathrm{~d} W_{t}
$$

for some random volatility vector $\left(\sigma_{t}^{j}\right)_{0 \leq t \leq T}:=\left(\sigma_{t}^{j, 1}, \cdots, \sigma_{t}^{j, q}\right)_{0 \leq t \leq T}$.

In view of Proposition 2.2, the first correction term $Y^{(1)}$ in the first order expansion $\bar{Y}_{t}^{\lambda, 1}=Y_{t}^{0}+\lambda Y_{t}^{(1)}$ is given by

$$
Y_{t}^{(1)}=-\mathbb{E}\left[\int_{t}^{T} e^{-\int_{t}^{s} r_{u} \mathrm{~d} u}(1-R)\left(Y_{s}^{0}\right)_{+} \mathrm{d} s \mid \mathcal{F}_{t}\right] .
$$

Remark 4.7. Note that, in the cases $\xi \leq 0$ and $\xi \geq 0$, we have $Y_{t}=Y_{t}^{0}$ and $Y_{t}=$ $e^{-\lambda(1-R)(T-t)} Y_{t}^{0}$ respectively (this results from an application of the comparison for BSDEs EPQ97, Theorem 2.2]). On the other hand, by [4.19), our 1-st order approximation reads as $\bar{Y}_{t}^{\lambda, 1}=Y_{t}^{0}$ and $\bar{Y}_{t}^{\lambda, 1}=(1-\lambda(1-R)(T-t)) Y_{t}^{0}$ respectively. Therefore, in terms of accuracy, the best setting for our 1-st order approx. is when the investor's position is almost surely negative $(\xi \leq 0)$, i.e. the true value $Y$ coincides with the proxy value $Y^{0}$. On the contrary, the worst setting seems to be when the investor's position is almost surely positive $(\xi \geq 0)$. In particular, in the latter case we have

$$
\operatorname{Err}_{t}^{\mathrm{Rel}}:=\frac{\left|Y_{t}-\bar{Y}_{t}^{\lambda, 1}\right|}{\left|Y_{t}\right|}=\frac{Y_{t}-\bar{Y}_{t}^{\lambda, 1}}{Y_{t}}=\frac{e^{-\lambda(1-R)(T-t)}-1+\lambda(1-R)(T-t)}{e^{-\lambda(1-R)(T-t)}} .
$$

This suggests that the first order expansion $\bar{Y}^{\lambda, 1}$ represents a good approximation for this task in terms of accuracy, which is fully coherent with Theorem 2.4. see also Figure 1 where the relative error 4.20), in the limiting case $\xi \geq 0$, is plotted as a function of the default intensity $\lambda$.



Figure 1: Relative error, as a function of the default intensity $\lambda$, of the 1-st order approximation $\bar{Y}_{0}^{\lambda, 1}$ in the limiting case $\xi \geq 0$. Here $R=0, t=0$ and $T=1$.

In order to compute the correcting term $Y^{(1)}$ in $(4.19)$, one option is to perform a Monte Carlo simulation of the proxy process $Y^{0}$ in 4.18), but this is not in the spirit of this paper. Alternatively, in the next subsection, we handle the analytical evaluation of the above conditional expectation (4.19), which is the corner stone to have a fully implementable numerical procedure. 


\subsection{Linearization and change of numeraire to compute $\bar{Y}^{\lambda, 1}$}

We use two rather standard techniques consisting in linearizing the positive part and applying suitable changes of numeraire. It gives

$$
Y_{t}^{(1)}=-(1-R) \sum_{j \in \mathcal{J}} c_{j} \int_{t}^{T} Y_{t, s}^{(1), j} \mathrm{~d} s, \quad Y_{t, s}^{(1), j}=\mathbb{E}\left[e^{-\int_{t}^{s} r_{u} \mathrm{~d} u} \Psi_{s}^{j} \mathbb{1}_{Y_{s}^{0} \geq 0} \mid \mathcal{F}_{t}\right] .
$$

Owing to the $\mathbb{Q}$-martingale and positive properties of $e^{-\int_{0}^{t} r_{s} \mathrm{~d} s} \Psi_{t}^{j}$ (since $\Psi^{j} \geq \mu^{j}>0$ ), it can be used as a numeraire to define a new probability measure (see [MR05]); namely, set

$$
\left.\frac{\mathrm{d} \mathbb{Q}^{j}}{\mathrm{~d} \mathbb{Q}}\right|_{\mathcal{F}_{t}}=L_{t}^{j}:=\frac{e^{-\int_{0}^{t} r_{s} \mathrm{~d} s} \Psi_{t}^{j}}{\Psi_{0}^{j}}
$$

Now, by applying the Bayes rule for change of numeraire we obtain

$$
Y_{t, s}^{(1), j}=\mathbb{E}_{\mathbb{Q}^{j}}\left[e^{-\int_{t}^{s} r_{u} \mathrm{~d} u} \Psi_{s}^{j} \mathbb{1}_{Y_{s}^{0} \geq 0} \frac{L_{t}^{j}}{L_{s}^{j}} \mid \mathcal{F}_{t}\right]=\Psi_{t}^{j} \mathbb{Q}^{j}\left(Y_{s}^{0} \geq 0 \mid \mathcal{F}_{t}\right) .
$$

It remains to compute the above conditional probabilities. We first observe that the processes $W_{t}^{j}=W_{t}-\int_{0}^{t}\left(\sigma_{s}^{j}\right)^{\top} \mathrm{d} s$ is a $q$-dimensional Brownian motion under the new measure $\mathbb{Q}^{j}$. The key point is to consider all prices in the new numeraire $e^{-\int_{0} r_{s} d s} \Psi^{j}$; indeed, the properties of change of numeraire ensure that $\left(\frac{\Psi_{s}^{i}}{\Psi_{s}^{j}}\right)_{s \geq 0}$ are exponential $\mathbb{Q}^{j}$-martingales with volatility vector $\sigma_{s}^{i}-\sigma_{s}^{j}$. Therefore we have

$$
\left\{Y_{s}^{0} \geq 0\right\}=\left\{\sum_{i \in \mathcal{J}}^{n} c_{i} \frac{\Psi_{s}^{i}}{\Psi_{s}^{j}} \geq 0\right\}=\left\{N_{s}^{j,+} \geq N_{s}^{j,-}\right\},
$$

with

$$
N_{s}^{j,+}=\sum_{i \in \mathcal{J}}\left(c_{i}\right)_{+} \frac{\Psi_{s}^{i}}{\Psi_{s}^{j}}, \quad N_{s}^{j,-}=\sum_{i \in \mathcal{J}}\left(c_{i}\right)_{-} \frac{\Psi_{s}^{i}}{\Psi_{s}^{j}}
$$

being positive martingales under $\mathbb{Q}^{j}$. To compute the conditional $\mathbb{Q}^{j}$-probability of the event $\left\{N_{s}^{j,+} \geq N_{s}^{j,-}\right\}$, we then follow the methodology inspired by GM14 using two approximations:

- the arithmetic means $N_{s}^{j,+}$ and $N_{s}^{j,-}$ are replaced by the geometric means, whose drift is modified to maintain the centering property.

- the volatility $\left(\sigma_{s}^{i}\right)_{s \geq t}$ is approximated by a proxy $\left(\bar{\sigma}_{t, s}^{i}\right)_{s \geq t}$ which is a square-integrable function w.r.t. $s$ and $\mathcal{F}_{t}$-measurable. The choice of the proxy depends on the context (local volatility or stochastic volatility model), see the discussion in Section 4.4 .

We define $M_{s}^{j,+}=\frac{N_{s}^{j,+}}{N_{t}^{j,+}}$ and $M_{s}^{j,-}=\frac{N_{s}^{j,-}}{N_{t}^{j,-}}$. The process $\left(M_{s}^{j,+}\right)_{s \geq t}$ is a positive martingale starting from 1 , whose dynamics is

$$
\mathrm{d} M_{s}^{j,+}=\frac{\sum_{i \in \mathcal{J}}\left(c_{i}\right)_{+} \mathrm{d} \frac{\Psi_{s}^{i}}{\Psi_{s}^{j}}}{N_{t}^{j,+}}=\frac{\sum_{i \in \mathcal{J}}\left(c_{i}\right)_{+}+\frac{\Psi_{s}^{i}}{\Psi_{s}^{j}}\left(\sigma_{s}^{i}-\sigma_{s}^{j}\right) \mathrm{d} W_{s}^{j}}{N_{t}^{j,+}} .
$$

Now, when $s$ is close to $t$ and/or volatilities are small, we have $M_{s}^{j,+} \approx 1$ and $\frac{\Psi_{s}^{i}}{\Psi_{s}^{j}} \approx \frac{\Psi_{t}^{i}}{\Psi_{t}^{j}}$, and thus

$$
\mathrm{d} M_{s}^{j,+} \approx M_{s}^{j,+} \frac{\sum_{i \in \mathcal{J}}\left(c_{i}\right)+\frac{\Psi_{t}^{i}}{\Psi_{t}^{j}}\left(\sigma_{s}^{i}-\sigma_{s}^{j}\right) \mathrm{d} W_{s}^{j}}{N_{t}^{j,+}}
$$




$$
\left.\approx M_{s}^{j,+} \frac{\sum_{i \in \mathcal{J}}\left(c_{i}\right)+\frac{\Psi_{t}^{i}}{\Psi_{t}^{j}}\left(\bar{\sigma}_{t, s}^{i}-\bar{\sigma}_{t, s}^{j}\right) \mathrm{d} W_{s}^{j}}{N_{t}^{j,+}} \quad \text { (by replacing } \sigma_{s}^{i}-\sigma_{s}^{j} \text { with } \bar{\sigma}_{t, s}^{i}-\bar{\sigma}_{t, s}^{j}\right) .
$$

We proceed analogously with $M^{j,-}$ and we obtain

$$
\begin{array}{r}
\left\{Y_{s}^{0} \geq 0\right\}=\left\{N_{t}^{j,+} M_{s}^{j,+} \geq N_{t}^{j,-} M_{s}^{j,-}\right\} \approx\left\{N_{t}^{j,+} \exp \left(\int_{t}^{s} \sigma_{t, r}^{j,+} \mathrm{d} W_{r}^{j}-\frac{1}{2} \int_{t}^{s}\left|\sigma_{t, r}^{j,+}\right|^{2} \mathrm{~d} r\right)\right. \\
\left.\geq N_{t}^{j,-} \exp \left(\int_{t}^{s} \sigma_{t, r}^{j,-} \mathrm{d} W_{r}^{j}-\frac{1}{2} \int_{t}^{s}\left|\sigma_{t, r}^{j,-}\right|^{2} \mathrm{~d} r\right)\right\}, \\
\text { where } \sigma_{t, s}^{j,+}:=\frac{\sum_{i \in \mathcal{J}}\left(c_{i}\right)_{+}+\frac{\Psi_{t}^{i}}{\Psi_{t}^{j}}\left(\bar{\sigma}_{t, s}^{i}-\bar{\sigma}_{t, s}^{j}\right)}{N_{t}^{j,+}}, \sigma_{t, s}^{j,-}:=\frac{\sum_{i \in \mathcal{J}}\left(c_{i}\right)-\frac{\Psi_{t}^{i}}{\Psi_{t}^{j}}\left(\bar{\sigma}_{t, s}^{i}-\bar{\sigma}_{t, s}^{j}\right)}{N_{t}^{j,-}} . \text { Finally, since }
\end{array}
$$
the proxy volatilities $\left(\bar{\sigma}_{t, s}^{i}\right)_{s>t}$ are $\mathcal{F}_{t}$-measurable, the $\mathbb{Q}^{j}$-conditional probabilities can be computed via Gaussian computations, i.e.

$$
\mathbb{Q}^{j}\left(Y_{s}^{0} \geq 0 \mid \mathcal{F}_{t}\right) \approx \mathcal{N}\left(\frac{\log \left(N_{t}^{j,+} / N_{t}^{j,-}\right)-\frac{1}{2} \int_{t}^{s}\left(\left|\sigma_{t, r}^{j,+}\right|^{2}-\left|\sigma_{t, r}^{j,-}\right|^{2}\right) \mathrm{d} r}{\sqrt{\int_{t}^{s}\left|\sigma_{t, r}^{j,+}-\sigma_{t, r}^{j,-}\right|^{2} \mathrm{~d} r}}\right) .
$$

It is justified in [GM14, that the above approximation on $Y_{s}^{0}$ is accurate in $L_{p}$ as the volatility and/or the time $s-t$ are small. In the same reference, additional explicit corrections are provided to the computation of the expectation, but we argue that this is unnecessary for the first-order expansion of this BSDE, because the approximation on $Y_{t}^{(1)}$ will be multiplied by the smaller factor $\lambda=\varepsilon$. Thus the neglected correction terms have no contributions at the first-order expansion. As explained in Avi09, $L_{p}$-approximations of $Y_{s}^{0}$ also provide approximations of its distribution function: we do not elaborate further in this direction since the computations are tedious and the result quite clear.

To sum up, our first order expansion for the solution of 4.16) is

$$
Y_{t} \approx \bar{Y}^{1}=Y_{t}^{0}+\lambda Y_{t}^{(1)},
$$

where the first order correcting term $Y^{(1)}$ is then approximated as

$$
Y_{t}^{(1)} \approx \tilde{Y}_{t}^{(1)}:=-(1-R) \sum_{j \in \mathcal{J}} c_{j} \Psi_{t}^{j} \int_{t}^{T} \mathcal{N}\left(\frac{\log \left(N_{t}^{j,+} / N_{t}^{j,-}\right)+\frac{1}{2} \int_{t}^{s}\left(\left|\sigma_{t, r}^{j,-}\right|^{2}-\left|\sigma_{t, r}^{j,+}\right|^{2}\right) \mathrm{d} r}{\sqrt{\int_{t}^{s}\left|\sigma_{t, r}^{j,-}-\sigma_{t, r}^{j,+}\right|^{2} \mathrm{~d} r}}\right) \mathrm{d} s .
$$

Remark 4.8. From practical point of view, the representation 4.22 for $\tilde{Y}^{(1)}$ can be considered semi-explicit. As such, given the explicit knowledge of the solution $Y^{0}$ of the linear problem (4.18), and of the proxy volatilities $\left(\bar{\sigma}_{t, s}^{j}\right)_{s \geq t}$, the above computation only requires a numerical integration with respect to the time-variables $r$ and $s$. It is important to observe that the dimension of the problem has no impact on the dimension of the numerical integral. Indeed, all the correlation between the prices is contained within the $q$-dimensional vectors $\sigma^{j,+}$ and $\sigma^{j,-}$, whereas the integrands in 4.22 are all 1-dimensional. In particular, a direct computation shows the following useful representation:

$$
\begin{aligned}
\left|\sigma_{t, s}^{j,+}\right|^{2} & =\frac{\sum_{i, k \in \mathcal{J}}\left(c_{i}\right)_{+}\left(c_{k}\right)_{+} \Psi_{t}^{i} \Psi_{t}^{k}\left(\Sigma_{t, s}^{j, j}+\Sigma_{t, s}^{i, k}-\Sigma_{t, s}^{j, i}-\Sigma_{t, s}^{j, k}\right)}{\left(\Psi_{t}^{j} N_{t}^{j,+}\right)^{2}}, \\
\left|\sigma_{t, s}^{j,-}\right|^{2} & =\frac{\sum_{i, k \in \mathcal{J}}\left(c_{i}\right)_{-}\left(c_{k}\right)_{-} \Psi_{t}^{i} \Psi_{t}^{k}\left(\Sigma_{t, s}^{j, j}+\Sigma_{t, s}^{i, k}-\Sigma_{t, s}^{j, i}-\Sigma_{t, s}^{j, k}\right)}{\left(\Psi_{t}^{j} N_{t}^{j,-}\right)^{2}},
\end{aligned}
$$




$$
\left|\sigma_{t, s}^{j,+}-\sigma_{t, s}^{j,-}\right|^{2}=\frac{\sum_{i, k \in \mathcal{J}}\left(\frac{\left(c_{i}\right)_{+}}{N_{t}^{j,+}}-\frac{\left(c_{i}\right)_{-}}{N_{t}^{j,-}}\right)\left(\frac{\left(c_{k}\right)_{+}}{N_{t}^{j,+}}-\frac{\left(c_{k}\right)_{-}}{N_{t}^{j,-}}\right) \Psi_{t}^{i} \Psi_{t}^{k}\left(\Sigma_{t, s}^{j, j}+\Sigma_{t, s}^{i, k}-\Sigma_{t, s}^{j, i}-\Sigma_{t, s}^{j, k}\right)}{\left(\Psi_{t}^{j}\right)^{2}},
$$

where $\Sigma=\left(\Sigma_{t, s}^{i, j}\right)_{i, j \in \mathcal{J}}$, with $\Sigma_{t, s}^{i, j}=\bar{\sigma}_{t, s}^{i} \cdot \bar{\sigma}_{t, s}^{j}$.

In the next subsection we present a possible choice for the proxy volatilities $\left(\bar{\sigma}_{t, s}^{j}\right)_{s \geq t}$ in the Markovian case.

\subsection{Log-normal volatility proxy $\bar{\sigma}^{j}$}

Let us consider a Markovian model driven by $d$ factors $X^{1}, \cdots, X^{d}$ of uncertainty (e.g. asset prices, indexes, volatilities etc) following the dynamics:

$$
\mathrm{d} X_{t}=\alpha\left(t, X_{t}\right) \mathrm{d} t+\beta\left(t, X_{t}\right) \mathrm{d} W_{t},
$$

with $\beta:[0, T] \times \mathbb{R}^{d} \rightarrow \mathbb{R}^{d \times q}$ and $\alpha:[0, T] \times \mathbb{R}^{d} \rightarrow \mathbb{R}^{d}$. Assume also a Markovian interest rate $r:[0, T] \times \mathbb{R}^{d} \rightarrow \mathbb{R}$, and Markovian terminal payoffs $\Psi^{j}=\psi^{j}\left(X_{T}\right), j \in \mathcal{J}$. Then, $Y_{t}^{0}$ is given by 4.18 with

$$
\Psi_{t}^{j}=u^{j}\left(t, X_{t}\right), \quad u^{j}(t, x)=\mathbb{E}\left[e^{-\int_{t}^{T} r\left(s, X_{s}\right) \mathrm{d} s} \psi^{j}\left(X_{T}\right) \mid X_{t}=x\right], \quad j \in \mathcal{J} .
$$

Now, by applying the Itô rule to $u^{j}\left(t, X_{t}\right)$ we get

$$
\sigma_{s}^{j}=\frac{\nabla_{x} u^{j}\left(s, X_{s}\right) \beta\left(s, X_{s}\right)}{u^{j}\left(s, X_{s}\right)}, \quad j \in \mathcal{J} .
$$

For any $t \geq 0$, we then define the $\mathcal{F}_{t}$-measurable approximations $\left(\bar{\sigma}_{t, s}^{j}\right)_{s \geq t}$ as

$$
\bar{\sigma}_{t, s}^{j}=\frac{\nabla_{x} u^{j}\left(s, X_{t}\right) \beta\left(s, X_{t}\right)}{u^{j}\left(s, X_{t}\right)}, \quad j \in \mathcal{J} .
$$

This approximation can be viewed as a log-normal approximation in the spirit of the shorttime asymptotic expansion proposed by LPP13, BG12. Further correcting terms could be computed and added to this approximation for $\sigma_{s}^{j}$, but we claim that this is not relevant in terms of accuracy due to the $\lambda$ term in 4.21), which is supposed to be relatively small.

\subsection{Example: Portfolio of Call and Put options}

\subsubsection{Notation}

As a particular example, we study the case where the global exposition of the investor with respect to his counterpart is given by a linear combination of call and put options written on $d$ underlying assets $S^{1}, \cdots, S^{d}$, whose dynamics are given by

$$
\mathrm{d} X_{t}^{i}=\left(r_{t}-\frac{1}{2}\left|\sigma_{t}^{i}\right|^{2}\right) \mathrm{d} t+\sigma_{t}^{i} \mathrm{~d} W_{t}, \quad i=1, \cdots, d,
$$

where $X_{t}^{i}:=\log \left(S_{t}^{i}\right), W$ is a $q$-dimensional Brownian motion with $q \geq d$, and $\left(\sigma_{t}^{i}\right)_{0 \leq t \leq T}$ are $\left(\mathbb{R}^{q}\right)^{\top}$-valued stochastic processes, square integrable with respect to $t$. This model allows for stochastic volatility and for stochastic interest rate $r$; therefore, $X$ may be non Markovian. Even though the analysis of the previous section applies to the case of a pool of derivatives with different maturities, we consider here for simplicity a portfolio of call and put options with the same maturity $T$, i.e. $\xi=\psi\left(X_{T}\right)$ with

$$
\psi(x)=\sum_{i=1}^{d} \sum_{l=1}^{n_{i}}\left[c_{C, i, l}\left(e^{x_{i}}-e^{k_{i, l}}\right)_{+}+c_{P, i, l}\left(e^{k_{i, l}}-e^{x_{i}}\right)_{+}\right]
$$




$$
=\sum_{i=1}^{d} \sum_{l=1}^{n_{i}}\left[c_{C, i, l}\left(e^{x_{i}} \vee e^{k_{i, l}}\right)+c_{P, i, l}\left(e^{k_{i, l}} \vee\left(2 e^{k_{i, l}}-e^{x_{i}}\right)\right)\right]-\sum_{i=1}^{d} \sum_{l=1}^{n_{i}}\left(c_{C, i, l}+c_{P, i, l}\right) e^{k_{i, l}},
$$

with some portfolio weights $c_{C, i, l}, c_{P, i, l} \in \mathbb{R}$. To fit our previous notation 4.17) one can set

$$
\mathcal{J}:=\left\{(\alpha, i, l) \mid \alpha \in\{C, P\}, 1 \leq i \leq d, 1 \leq l \leq n_{i}\right\} \cup \Delta,
$$

and

$$
\Psi^{(C, i, l)}=e^{k_{i, j}} \vee e^{X_{T}^{i}}, \quad \Psi^{(P, i, l)}=e^{k_{i, l}} \vee\left(2 e^{k_{i, l}}-e^{X_{T}^{i}}\right), \quad 1 \leq i \leq d, \quad 1 \leq l \leq n_{i},
$$

together with

$$
\Psi^{\Delta}=1, \quad c_{\Delta}=-\sum_{i=1}^{d} \sum_{l=1}^{n_{i}}\left(c_{C, i, l}+c_{P, i, l}\right) e^{k_{i, l}} .
$$

\subsubsection{Numerical tests under a multidimensional constant volatility model}

We consider the particular case of constant parameters dynamics for the underlying assets, i.e. 4.24 reduces to $\sigma^{i} \cdot \sigma^{j}=\left|\sigma^{i}\right|\left|\sigma^{j}\right| \rho_{i, j}$ where $\rho_{i, j}$ stands for the correlation between the $i$-th and $j$-th assets.

To the best of our knowledge there are no benchmarks in literature for the valuation of the FVA with provision of counterparty risk in such a multidimensional framework. The implementation of a Monte Carlo simulation for the evaluation of $Y_{t}$ would be quite challenging and goes far beyond the purpose of this paper. Therefore, we rely on the error estimate (2.5), and on Remark 4.7. to claim that the first order expansion $\bar{Y}^{1, \lambda}$ represents an accurate approximation for $\lambda$ reasonably small, say $\lambda<5 \%$.

In the following numerical tests we consider the approximated first order expansion $\tilde{\bar{Y}}_{t}^{1, \lambda}=Y_{t}^{0}+\lambda \tilde{Y}_{t}^{(1)}$, where $\tilde{Y}^{1}$ is then given by 4.22 -4.21 -4.23, with

$$
\Psi_{t}^{\Delta}=e^{-r(T-t)}, \quad \Sigma_{t, s}^{\Delta, \zeta}=\Sigma_{t, s}^{\zeta, \Delta}=0, \quad \zeta \in \mathcal{J},
$$

and with

$$
\begin{aligned}
\Psi_{t}^{\eta} & =\Psi_{t}^{(\alpha, i, j)}=B S^{\alpha, i, j}\left(t, X_{t}^{i}\right)+e^{k_{i, j}-r(T-t)} \\
\Sigma_{t, s}^{\eta, \zeta} & =\bar{\sigma}_{t, s}^{\eta} \cdot \bar{\sigma}_{t, s}^{\zeta}=\bar{\sigma}_{t, s}^{(\alpha, i, j)} \cdot \bar{\sigma}_{t, s}^{(\beta, k, l)} \\
& =\left|\sigma_{i}\right|\left|\sigma_{k}\right| \rho_{i, k} \frac{\partial_{x_{i}} B S^{\alpha, i, j}\left(s, X_{t}^{i}\right) \partial_{x_{k}} B S^{\beta, k, l}\left(s, X_{t}^{k}\right)}{\left(B S^{\alpha, i, j}\left(s, X_{t}^{i}\right)+e^{k_{i, j}-r(T-s)}\right)\left(B S^{\beta, k, l}\left(s, X_{t}^{k}\right)+e^{k_{k, l}-r(T-s)}\right)},
\end{aligned}
$$

for any $\eta=(\alpha, i, j), \zeta=(\beta, k, l)$, with $\alpha, \beta \in\{C, P\}, 1 \leq i, k \leq d, 1 \leq j \leq n_{i}, 1 \leq l \leq n_{k}$, and where $\mathrm{BS}^{C, i, j}\left(t, X_{t}^{i}\right)$ and $\mathrm{BS}^{P, i, j}\left(t, X_{t}^{i}\right)$ are the usual prices at time $t$ of a Call and Put options respectively, with log-strike $k_{i, j}$, under the Black-Scholes model with volatility $\sigma_{i}$ and interest rate $r$. Note that, to compute 4.25) we used the time-dependent approximation discussed at the end of the previous section, which now reads as

$$
\bar{\sigma}_{t, s}^{\eta}=\bar{\sigma}_{t, s}^{\alpha, i, j}=\frac{\partial_{x^{i}} \mathrm{BS}^{(\alpha, i, j)}\left(s, X_{t}^{i}\right) \sigma_{i}}{\operatorname{BS}^{\alpha, i, j}\left(s, X_{t}^{i}\right)+e^{k_{i, j}-r(T-s)}} .
$$

1-st numerical test: 5 underlying assets. We consider a portfolio with 5 options (Put or Call), each of these being written on a different underlying asset, and we compare the the semi-explicit 1-st order approximation $\tilde{\bar{Y}}^{1, \lambda}$ with the former first order approximation $\bar{Y}^{1, \lambda}$, computed by means of Monte Carlo simulation. The correlation is set to $\rho_{i, j}=0$ $(i \neq j)$, the interest rate is $r=5 \%$ and the default intensity is $\lambda=4 \%$. The remaining 
parameters are chosen according to two different scenarios summarized in Tables 3 and 4 , and their corresponding results are reported in Tables 5 and 6 respectively. In particular, the first two columns represent the value of the portfolio at time 0 with terminal payoff $\xi^{-}$ and $\xi^{+}$respectively, the third column represents the 0 -th order approximation $Y_{0}^{0}$ of $Y_{0}$. Note that $Y^{0}$ is the solution of the linear BSDE (4.16) with $\lambda=0$, and thus $Y^{0}=Y^{+}-Y^{-}$. The fourth column contains the semi-closed 1-st order approx. $\tilde{\bar{Y}}_{0}^{1, \lambda}$, whereas the fifth one contains the extreme values of the $95 \%$ confidence interval of the Monte Carlo simulations for the 1-st order approx. $\bar{Y}_{0}^{1, \lambda}$. We observe that the proxy approximation of volatilities do not deteriorate significantly the results (see the difference between $\tilde{\bar{Y}}_{0}^{1, \lambda}$ and $\bar{Y}_{0}^{1, \lambda}$ ). The amplitude of the correction terms seemingly increases as $T$ increases, which is somehow coherent with estimates w.r.t. $T$ in Theorem 2.4

2-nd numerical test: 10 underlying assets. In our second numerical test we consider a portfolio composed by 10 Call and Put options, each of them being written on a different underlying asset. The main purpose of this experiment is to show the flexibility of our methods. In particular, to show how this easily adapts to the dimensional increment, and then it could be thought as a viable avenue to the purpose of computing the FVA for a multiasset portfolio. On the contrary, the regression Monte-Carlo scheme GT13b can handle more general BSDEs but it suffers from the curse of dimensionality; indeed, the theoretical order of convergence w.r.t. the computational effort is $(8+d / k)^{-1}$ where $d$ is the dimension and $k$ the regularity of the value fonction to compute.

We assume once again no correlation between assets, we set the interest rate to $r=5 \%$ and the default intensity to $\lambda=3 \%$. The choice of the remaining parameters is summarized in Table 7. In Figures 2, 3 and 4 we show, for $T=0.2, T=1$ and $T=2$ respectively, how the portfolio value (with and without FVA) changes, as the volatilities $\left(\sigma_{i}\right)_{0 \leq i \leq 10}$ are multiplied by a factor $v$. In particular the solid blue and the dashed red lines represent, respectively, the proxy value $Y_{0}^{0}$, i.e. the portfolio value without FVA, and the first order approximation $\tilde{\bar{Y}}_{0}^{1, \lambda}$ of $Y_{0}$, i.e. the approximate portfolio value with provision for counterparty risk, both plotted as a function of $v \in[0.5,2]$.

\begin{tabular}{c|ccccc}
\hline \hline Underlying asset & 1 & 2 & 3 & 4 & 5 \\
\hline$\sigma$ & 0.2 & 0.3 & 0.1 & 0.3 & 0.2 \\
\hline$k$ & -0.1 & -0.2 & 0 & -0.1 & 0.1 \\
\hline Call options & 1 & 0 & -1 & 0 & 1 \\
\hline Put options & 0 & -1 & 0 & 1 & 0 \\
\hline \hline
\end{tabular}

Table 3: Parameters in the Scenario 1.A

\begin{tabular}{c|ccccc}
\hline \hline Underlying asset & 1 & 2 & 3 & 4 & 5 \\
\hline$\sigma$ & 0.2 & 0.2 & 0.3 & 0.1 & 0.2 \\
\hline$k$ & 0.3 & 0 & -0.1 & -0.3 & 0.2 \\
\hline Call options & 0 & 1 & 1 & -1 & 0 \\
\hline Put options & -1 & 0 & 0 & 0 & 1 \\
\hline \hline
\end{tabular}

Table 4: Parameters in the Scenario 1.B 


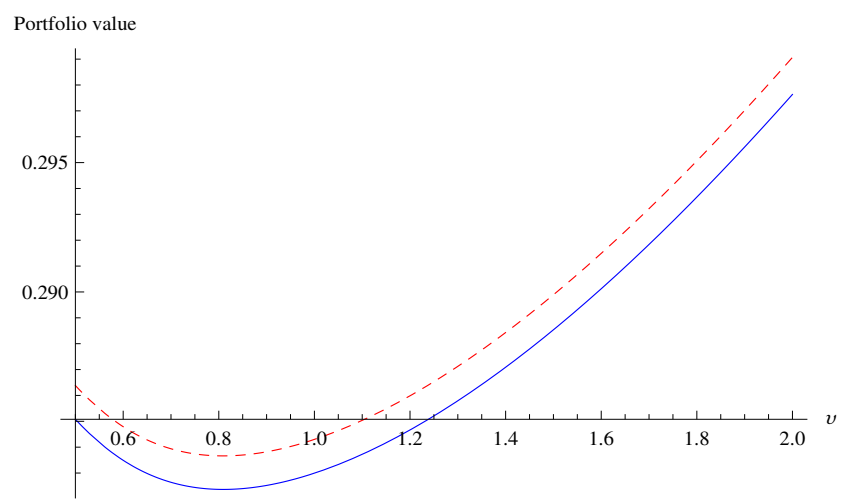

Figure 2: $T=0.2$. Portfolio value with (solid line) and without (dashed line) FVA, as a function of $v$.

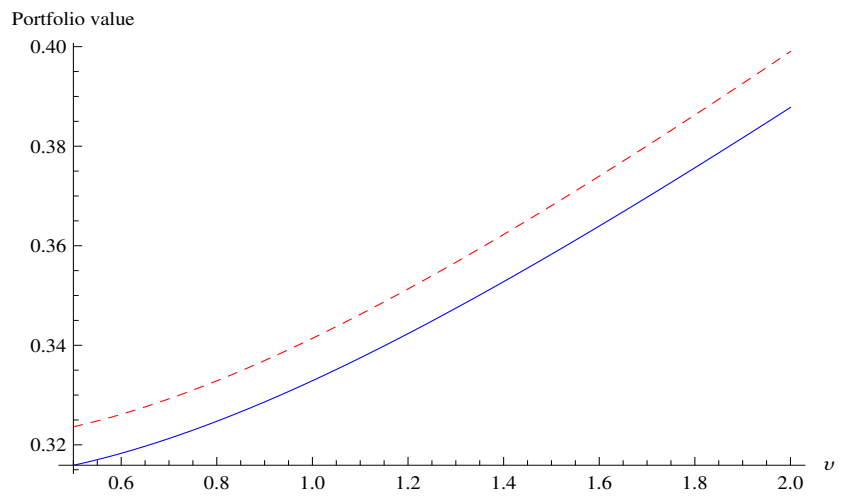

Figure 3: $T=1$. Portfolio value with (solid line) and without (dashed line) FVA, as a function of $v$.

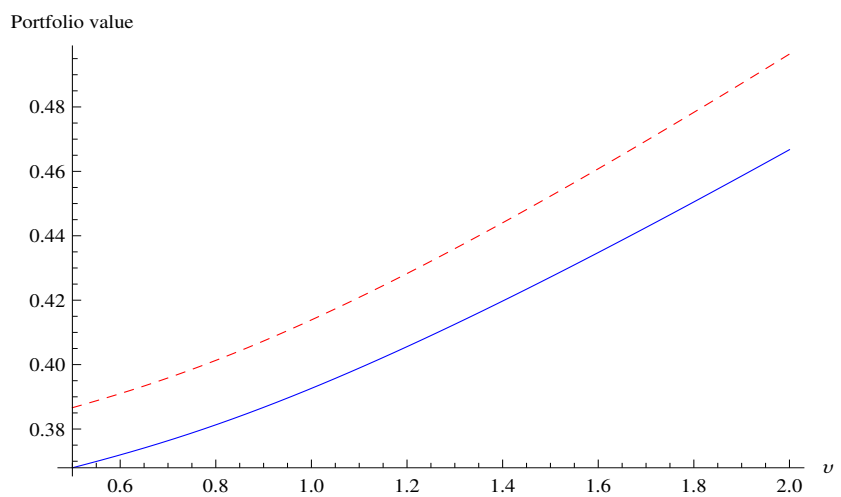

Figure 4: $T=2$. Portfolio value with (solid line) and without (dashed line) FVA, as a function of $v$. 


\begin{tabular}{|c|ccccc|}
\hline$T$ & $Y_{0}^{-}$ & $Y_{0}^{+}$ & $Y_{0}^{0}=Y_{0}^{+}-Y_{0}^{-}$ & $\tilde{Y}_{0}^{1, \lambda}$ & $\bar{Y}_{0}^{1, \lambda}(\mathrm{MC} 95 \%$ C.I. $)$ \\
\hline \hline 0.1 & 0.1285 & 0.0000 & 0.0932 & 0.0930 & $0.0930-0.0930$ \\
\hline 0.5 & 0.1958 & 0.0080 & 0.1370 & 0.1353 & $0.1353-0.1353$ \\
\hline 1 & 0.2498 & 0.0320 & 0.1790 & 0.1745 & $0.1745-0.1745$ \\
\hline 1.5 & 0.2912 & 0.0611 & 0.2133 & 0.2053 & $0.2052-0.2052$ \\
\hline 2 & 0.3259 & 0.0913 & 0.2431 & 0.2308 & $0.2306-0.2307$ \\
\hline
\end{tabular}

Table 5: Comparison in the Scenario 1.A.

\begin{tabular}{|c|ccccc|}
\hline$T$ & $Y_{0}^{-}$ & $Y_{0}^{+}$ & $Y_{0}^{0}=Y_{0}^{+}-Y_{0}^{-}$ & $\tilde{Y}_{0}^{1, \lambda}$ & $\bar{Y}_{0}^{1, \lambda}(\mathrm{MC} 95 \%$ C.I. $)$ \\
\hline \hline 0.1 & 0.6060 & 0.3487 & -0.2572 & -0.2572 & $-0.2572--0.2572$ \\
\hline 0.5 & 0.5956 & 0.4195 & -0.1759 & -0.1761 & $-0.1761--0.1761$ \\
\hline 1 & 0.5908 & 0.4863 & -0.1022 & -0.1036 & $-0.1037--0.1036$ \\
\hline 1.5 & 0.5915 & 0.5419 & -0.0444 & -0.0478 & $-0.0479--0.0479$ \\
\hline 2 & 0.5950 & 0.5905 & 0.0042 & -0.0021 & $-0.0023--0.0022$ \\
\hline
\end{tabular}

Table 6: Comparison in the Scenario 1.B

\section{A. Appendix}

\section{A.1. A priori estimates of the solutions of BSDE's}

For the convenience of the reader, we recall the following standard stability result.

Proposition A.1. EPQ97, Proposition 2.1 and second line p.20] Let $\left(\tilde{Y}^{i}, \tilde{Z}^{i}\right)(i=1,2)$ be the solutions of the BSDEs respectively associated to the terminal conditions $\tilde{\xi}^{i} \in \mathbb{L}_{T}^{2}(\mathbb{R})$ and to the drivers $\tilde{f}^{i}$, which are uniformly Lipschitz in $(y, z)$ and such that $\tilde{f}^{i}(., 0,0) \in \mathbb{H}_{T}^{2}(\mathbb{R})$. Set $\delta_{t}:=\tilde{f}^{1}\left(t, \tilde{Y}_{t}^{2}, \tilde{Z}_{t}^{2}\right)-\tilde{f}^{2}\left(t, \tilde{Y}_{t}^{2}, \tilde{Z}_{t}^{2}\right)$ and denote by $\tilde{C}_{1}$ the Lipschitz constant for $\tilde{f}^{1}$. Then, for any $(\lambda, \mu, \theta)$ such that $\mu>0, \lambda^{2}>\tilde{C}_{1}$ and $\theta \geq \tilde{C}_{1}\left(2+\lambda^{2}\right)+\mu^{2}$, we have

$$
\begin{aligned}
\sup _{0 \leq t \leq T} e^{\theta t}\left|\tilde{Y}_{t}^{1}-\tilde{Y}_{t}^{2}\right|_{L_{2}}^{2} & \leq e^{\theta T}\left|\tilde{\xi}^{1}-\tilde{\xi}^{2}\right|_{L_{2}}^{2}+\frac{1}{\mu^{2}}\|\delta\|_{\theta}^{2}, \\
\left\|\tilde{Z}^{1}-\tilde{Z}^{2}\right\|_{\theta}^{2} & \leq \frac{\lambda^{2}}{\lambda^{2}-\tilde{C}_{1}}\left[e^{\theta T}\left|\tilde{\xi}^{1}-\tilde{\xi}^{2}\right|_{L_{2}}^{2}+\frac{1}{\mu^{2}}\|\delta\|_{\theta}^{2}\right] .
\end{aligned}
$$

\section{A.2. Proof of Proposition 1.1}

We set $G_{t}^{0}:=\eta_{t}^{Y} Y_{t}^{0}+\eta_{t}^{Z} \cdot Z_{t}^{0}$ and $G_{t}:=\eta_{t}^{Y} u_{t}+\eta_{t}^{Z} \cdot v_{t}$ : we aim at proving

$$
\mathcal{I}(\varepsilon):=\mathbb{E} \int_{0}^{T}\left|\frac{\left(G_{t}^{0}+\varepsilon G_{t}\right)_{+}-\left(G_{t}^{0}\right)_{+}-\varepsilon\left[G_{t} \mathbb{1}_{G_{t}^{0}>0}+\left(G_{t}\right)_{+} \mathbb{1}_{G_{t}^{0}=0}\right]}{\varepsilon}\right|^{2} \mathrm{~d} t \rightarrow 0
$$

as $\varepsilon \rightarrow 0^{+}$. Use $(a+b)_{+}-a_{+}=b \int_{0}^{1} \mathbb{1}_{a+\lambda b>0} \mathrm{~d} \lambda$ (for any $a, b \in \mathbb{R}$ ) and write

$$
\begin{aligned}
\mathcal{I}(\varepsilon) & =\mathbb{E} \int_{0}^{T}\left|G_{t} \int_{0}^{1} \mathbb{1}_{G_{t}^{0}+\lambda \varepsilon G_{t}>0} \mathrm{~d} \lambda-\left[G_{t} \mathbb{1}_{G_{t}^{0}>0}+\left(G_{t}\right)_{+} \mathbb{1}_{G_{t}^{0}=0}\right]\right|^{2} \mathrm{~d} t \\
& :=\mathcal{I}_{<}(\varepsilon)+\mathcal{I}_{>}(\varepsilon)+\mathcal{I}_{=}(\varepsilon)
\end{aligned}
$$

where $\mathcal{I}_{>,<,=}(\varepsilon)$ denote the above expectation-integral respectively on the sets $\left\{G_{t}^{0}<0\right\}$, $\left\{G_{t}^{0}>0\right\},\left\{G_{t}^{0}=0\right\}$. We have

$$
\begin{aligned}
\mathcal{I}_{<}(\varepsilon) & =\mathbb{E} \int_{0}^{T} \mathbb{1}_{G_{t}^{0}<0}\left|G_{t} \int_{0}^{1} \mathbb{1}_{G_{t}^{0}+\lambda \varepsilon G_{t}>0} \mathrm{~d} \lambda\right|^{2} \mathrm{~d} t \\
& =\mathbb{E} \int_{0}^{T} \int_{0}^{1} \int_{0}^{1} \mathbb{1}_{G_{t}^{0}<0} G_{t}^{2} \mathbb{1}_{G_{t}^{0}+\lambda_{2} \varepsilon G_{t}>0} \mathbb{1}_{G_{t}^{0}+\lambda_{1} \varepsilon G_{t}>0} \mathrm{~d} \lambda_{1} \mathrm{~d} \lambda_{2} \mathrm{~d} t .
\end{aligned}
$$




\begin{tabular}{c|cccccccccc}
\hline \hline Underlying asset & 1 & 2 & 3 & 4 & 5 & 6 & 7 & 8 & 9 & 10 \\
\hline$\sigma$ & 0.2 & 0.3 & 0.1 & 0.3 & 0.2 & 0.2 & 0.3 & 0.1 & 0.3 & 0.2 \\
\hline$k$ & -0.1 & -0.2 & 0 & -0.1 & 0.1 & -0.1 & -0.2 & 0 & -0.1 & 0.1 \\
\hline Call options & 1 & 0 & -1 & 0 & 1 & 1 & 0 & -1 & 0 & 1 \\
\hline Put options & 0 & -1 & 0 & 1 & 0 & 0 & -1 & 0 & 1 & 0 \\
\hline \hline
\end{tabular}

Table 7: Parameters in the Scenario 2

The integrand is bounded by $G^{2}$ which is $\mathrm{d} \mathbb{P} \times \mathrm{d} \lambda_{1} \times \mathrm{d} \lambda_{2} \times \mathrm{d} t$-integrable; furthermore, the integrand converges $\mathrm{d} \mathbb{P} \times \mathrm{d} \lambda_{1} \times \mathrm{d} \lambda_{2} \times \mathrm{d} t$-a.e. to 0 as $\varepsilon \rightarrow 0$. Thus, by the dominated convergence theorem, we have $\mathcal{I}_{<}(\varepsilon) \rightarrow 0$. Similarly, $\mathcal{I}_{>}(\varepsilon) \rightarrow 0$. Last, observe that $\mathcal{I}_{=}(\varepsilon)=$ $\mathbb{E} \int_{0}^{T} \mathbb{1}_{G_{t}^{0}=0}\left|G_{t} \int_{0}^{1} \mathbb{1}_{\lambda \varepsilon G_{t}>0} \mathrm{~d} \lambda-\left(G_{t}\right)_{+}\right|^{2} \mathrm{~d} t=0$ since $\varepsilon>0$. This concludes the proof.

\section{References}

\section{References}

[Avi09] R. Avikainen. On irregular functionals of SDEs and the Euler scheme. Finance and Stochastics, 13:381-401, 2009.

[BD07] C. Bender and R. Denk. A forward scheme for backward SDEs. Stochastic Process. Appl., 117(12):1793-1812, 2007.

[BG12] R. Bompis and E. Gobet. Asymptotic and non asymptotic approximations for option valuation. In T. Gerstner and P. Kloeden, editors, Recent Developments in Computational Finance: Foundations, Algorithms and Applications, chapter 4, pages 159-241. World Scientific Publishing Company, 2012.

[Cre13] S. Crepey. Financial modeling: a Backward Stochastic Differential Equations perspective. Springer. Springer-Verlag, Berlin, 2013.

[EHM08] N. El Karoui, S. Hamadène, and A. Matoussi. Backward stochastic differential equations and applications. In R. Carmona, editor, Indifference pricing: theory and applications, chapter 8, pages 267-320. Springer-Verlag, 2008.

[EPQ97] N. El Karoui, S. Peng, and M.C. Quenez. Backward stochastic differential equations in finance. Mathematical Finance, 7(1):1-71, 1997.

[GH14] J. Guyon and P. Henry-Labordère. Non linear pricing. CRC Financial Mathematics. Chapman and Hall, 2014.

[GLW05] E. Gobet, J.-P. Lemor, and X. Warin. A regression-based Monte Carlo method to solve backward stochastic differential equations. Ann. Appl. Probab., 15(3):2172$2202,2005$.

[GM14] E. Gobet and M. Miri. Weak approximation of averaged diffusion processes. Stochastic Processes and their Applications, 124:475-504, 2014.

[GT13a] E. Gobet and P. Turkedjiev. Approximation of BSDEs using Malliavin weights and least-squares regression. In revision for Bernoulli, 2013.

[GT13b] E. Gobet and P. Turkedjiev. Linear regression MDP scheme for discrete backward stochastic differential equations under general conditions. In revision for Mathematics of Computation, 2013. 
[HNS11] Y. Hu, D. Nualart, and X. Song. Malliavin calculus for backward stochastic differential equations and application to numerical solutions. Ann. Appl. Probab., 21(6):2379-2423, 2011.

[LPP13] M. Lorig, S. Pagliarani, and A. Pascucci. Explicit implied vols for multifactor local-stochastic vol models. ArXiv preprint arXiv:1306.5447, 2013.

[MR05] M. Musiela and M. Rutkowski. Martingale methods in financial modelling. Springer Verlag, second edition, 2005.

[PP14] S. Pagliarani and A. Pascucci. Asymptotic expansions for degenerate parabolic equations, http://papers.ssrn.com/sol3/papers.cfm?abstract_ id=2442774. Preprint SSRN, 2014.

[PR14] E. Pardoux and A. Rascanu. Stochastic Differential Equations, Backward SDEs, Partial Differential Equations, volume 69 of Stochastic Modelling and Applied Probability. Springer-Verlag, 2014.

[TY13] A. Takahashi and T. Yamada. On an asymptotic expansion of forward-backward SDEs with a perturbed driver, http://papers.ssrn.com/sol3/papers.cfm? abstract_id=2319358. Preprint SSRN, 2013. 